\title{
The role of tropopause polar vortices in the intensification of summer Arctic cyclones
}

\author{
Suzanne L. Gray ${ }^{1}$, Kevin I. Hodges ${ }^{1,2}$, Jonathan L. Vautrey ${ }^{1, *}$, and John Methven ${ }^{1}$ \\ ${ }^{1}$ Department of Meteorology, University of Reading, Reading, RG6 6ET, UK \\ ${ }^{2}$ National Centre for Atmospheric Science, University of Reading, Reading, RG6 6ET, UK \\ *Now at Met Office, Exeter, UK
}

Correspondence: Suzanne Gray (s.l.gray@ reading.ac.uk)

\begin{abstract}
Human activity in the Arctic is increasing as new regions become accessible, with a consequent need for improved understanding of hazardous weather there. Arctic cyclones are the major weather systems affecting the Arctic environment during summer, including the sea ice distribution. Meso- to synoptic-scale tropopause polar vortices (TPVs) frequently occur in polar regions and are a proposed mechanism for Arctic cyclone genesis and intensification. However, while the importance

5 of pre-existing tropopause-level features for cyclone development, and their existence as part of the three-dimensional mature cyclone structure, is well established in the mid-latitudes, evidence of the importance of pre-existing TPVs for Arctic cyclone development is mainly limited to a few case studies. Here we examine the extent to which Arctic cyclone growth is coupled to TPVs by analysing a climatology of summer Arctic cyclones and TPV characteristics produced by tracking both features in the latest ECMWF reanalysis (ERA5).

The annual counts of Arctic cyclones and TPVs are significantly correlated for features with genesis either within or outside the Arctic, implying that TPVs have a role in the development of Arctic cyclones. However, from their proximity, only about one third of Arctic cyclones intensify while influenced by a TPV and a maximum of $10 \%$ have a nearby TPV at their genesis time. Consistent with the track densities of the full sets of Arctic cyclones and TPVs, cyclones associated with TPVs during their intensification phase (matched cyclones) track preferentially over the Arctic Ocean along the North American coastline and Canadian Archipelago. In contrast, cyclones intensifying distant from any TPV (unmatched cyclones) track preferentially along the north coast of Eurasia. Composite analysis reveals the presence of a distinct relative vorticity maximum at and above the tropopause level associated with the TPV throughout the intensification period for matched cyclones and that these cyclones have a reduced upstream tilt compared to unmatched cyclones. Interaction of cyclones with TPVs has implications for the predictability of Arctic weather, given the long lifetime, but relatively small spatial scale of TPVs compared with the density of the polar observation network.
\end{abstract}

\section{Introduction}

Arctic cyclones are typically defined as synoptic-scale cyclones developing within, or moving into, the Arctic region. In summer, Arctic cyclones are larger than the polar meso-cyclones that are common during Arctic winter, of which polar lows are the most intense subset. Climate models project continued summer sea ice reductions in the Arctic with nearly ice-free conditions 
by the middle of this century (Overland and Wang, 2013). Human activity in the Arctic, such as from as shipping and offshore operations, is expected to increase as new regions become accessible (Stephenson et al., 2013) with a consequent need for reliable weather forecasts, particularly of hazardous weather. Arctic cyclones also locally affect sea ice cover through surface fluxes and wind forcing, with cyclones appearing to slow the general day-to-day decline in concentration during the summer months (Finocchio et al., 2020; Schreiber and Serreze, 2020). Arctic cyclones are projected to change as climate changes, with increases in dynamical intensity and frequency in summer associated with enhancement of the land-sea thermal contrast along the Arctic coastline termed the Arctic Frontal Zone (Day and Hodges, 2018), but reductions in cyclone intensity in winter (Day et al., 2014). Wickström et al. (2020) link recent historical (1979-2016) significant trends in winter cyclone densities in the Svalbard and Barents Sea regions to a change to a more meridional North Atlantic storm track. An ongoing World Meteorological Organisation World Weather Research Programme (the Polar Prediction Project) was developed in recognition of the importance of improved weather and environmental prediction services for the Polar Regions. The meso- to synoptic-scale tropopause-based coherent vortices (called tropopause polar vortices, TPVs) frequently observed in polar regions (Hakim and Canavan, 2005; Cavallo et al., 2009) are hypothesised to play a central role in Arctic cyclone genesis and intensification (Tao et al., 2017; Yamagami et al., 2017, 2018a). In this paper we present a climatology of summer Arctic cyclones and TPVs by tracking features in the latest global reanalysis of the European Centre for Medium-Range Weather Forecasts (ECMWF), ERA5, and deduce the role of TPVs in the initiation, intensification and structure of Arctic cyclones.

Arctic cyclones are often distinguished from their extratropical cousins by a simple latitude threshold. The climatological characteristics of Arctic cyclones (and indeed all cyclones) are somewhat dependent on the identification and tracking tool used for analysis as well as the dataset to which it is applied (typically a reanalysis). Using the National Centers for Environmental Prediction-National Center for Atmospheric Research (NCEP-NCAR) reanalysis (from 1948-2002) and tracking mean sea level pressure (MSLP) anomalies, Zhang et al. (2004) found the number of Arctic cyclones (cyclones north of $60^{\circ}$ ) increased slightly from winter to summer (from about 65 to 75 per month). The summer cyclones had slightly longer durations (exceeding 40 hours on average) but reduced intensity, measured by MSLP, compared to the winter cyclones. Cyclones generated locally in the Arctic region were weaker than those tracking into it from the mid-latitudes. Enhanced model resolution (and improved data assimilation) in reanalyses leads to an improved representation of cyclones north of $55^{\circ} \mathrm{N}$ with deeper central pressures, faster deepening, stronger winds and up to a $40 \%$ increase in summer (June-October) cyclone numbers when comparing the Arctic System reanalysis, which has $30 \mathrm{~km}$ horizontal grid spacing, with coarser resolution reanalyses (Tilinina et al., 2014). However, as expected, this difference in numbers is most marked for weaker cyclones (central MSLPs exceeding 980 $\mathrm{hPa}$ ). Also when comparing the reanalyses for just the Arctic Ocean region, the differences between them were much reduced with the Arctic System reanalysis having only 9\% more cyclones than the average of the other reanalyses considered. The analysed characteristics of Arctic cyclones depend on the feature identification method. Cyclone frequency was found to be higher in winter than summer when identifying synoptic-scale cyclones using $850 \mathrm{hPa}$ relative vorticity $\left(\xi_{850}\right)$, but similar when identifying them using MSLP using the same tracking algorithm (Vessey et al., 2020). These differences can exceed those arising from the use of different reanalyses. When identifying using $\xi_{850}$, about 100 synoptic-scale Arctic cyclones (cyclones north of $65^{\circ} \mathrm{N}$ ) occurred each summer season (June-August) and 120 each winter season (December-February) with 
about half of these cyclones having their genesis in the Arctic region and the other half tracking into it from the mid-latitudes in both seasons. In contrast, only about 65 cyclones occurred each summer and winter season when identifying them using MSLP. Contrary to the range in cyclone numbers, previous studies generally agree on the geographical characteristics of Arctic cyclones. In summer cyclones tend to track along the Arctic Frontal Zone region, particularly along the northern Eurasian coastline, and into the Arctic Ocean (e.g., Vessey et al., 2020). The east coast of Greenland and the northern Canadian coastline are also regions of cyclogenesis.

Tropopause polar vortices (TPVs) are defined by Cavallo et al. (2009) as long-lived coherent vortices associated with a meso- to synoptic-scale depression of the tropopause (most often less than $1000 \mathrm{~km}$ in radius). These can last longer than a month (Hakim and Canavan, 2005) and can be isolated from other features at tropopause level. Their importance is associated with their ability to spin up low-level disturbances through baroclinic interaction, with their longevity offering up potential for enhanced predictability. Note that TPVs are distinct from the much larger-scale tropospheric polar vortex and stratospheric winter polar vortex that are both associated with the reservoir of higher potential vorticity (PV) values occupying high latitudes. The PV gradient is often sharp on the edge of the tropospheric polar vortex, where isentropic surfaces intersect the tropopause, and the westerly jet streams are located on these sharp gradients. One source of confusion with the terminology used in the literature arises because the tropospheric polar vortex weakens (and its area shrinks) between winter and summer to such an extent that it eventually breaks up into a number of smaller cut-off PV features at tropopause level. After this happens, the individual features have been referred to as both polar vortices and TPVs, although they may be quite large in scale (especially in early summer). Eventually, the larger-scale tropospheric polar vortex is re-established in autumn as a result of increasing net radiative cooling re-building the PV reservoir.

Cyclonic TPVs are associated with a lowered tropopause and therefore larger values of PV where stratospheric air is at lower altitude than usual. The PV feature may be cut-off from other PV features on isentropic surfaces intersecting the tropopause, or it may be on the edge of a large-scale Rossby wave trough. Either way, the dynamic tropopause surface has a local minimum in altitude describing the centre of the TPV. Many studies, including ours, use the surface where the PV equals 2 PVU (potential vorticity units, $1 \mathrm{PVU}=10^{-6} \mathrm{~m}^{2} \mathrm{~s}^{-1} \mathrm{~K} \mathrm{~kg}^{-1}$ ) to define the dynamic tropopause; negative anomalies of potential temperature, $\theta$, on the 2 PVU surface are used to identify TPVs. Note that $\theta$ increases with height throughout most of the atmosphere, indicating stable stratification, so a TPV associated with a minimum in tropopause height must naturally be associated with a minimum in $\theta$ relative to its surroundings on the tropopause. In the Northern Hemisphere, the positive PV anomaly characterising the TPV is associated with positive $\xi$, and anticlockwise relative motion, as well as a local maximum in static stability $(\partial \theta / \partial z)$. If the TPV is an isolated PV feature, then this is achieved by vertically bunching isentropic surfaces towards the centre of the PV anomaly (just above the tropopause), resulting in regions with lower static stability above and below. The isentropic surfaces dip down in the lower stratosphere above the PV anomaly and therefore there is a positive $\theta$-anomaly $\left(\theta^{\prime}\right)$ there (and also high temperature anomaly relative to surroundings on each pressure surface). In the upper troposphere below the anomaly, isentropic surfaces bow upwards and there is a negative $\theta^{\prime}$ that is often described as a "cold core".

In Cavallo et al. (2010) composite analysis of TPVs forming in the Canadian Arctic region over a two-year period, simulated using the Weather Research and Forecasting (WRF) model, revealed peak temperature anomalies of $-8.5 \mathrm{~K}$ and $+5.5 \mathrm{~K}$ in 
https://doi.org/10.5194/wcd-2021-30

Preprint. Discussion started: 7 June 2021

(c) Author(s) 2021. CC BY 4.0 License.

Weather and

Climate Dynamics

Discussions

L.

the troposphere and above the tropopause (at around $250 \mathrm{hPa}$ ), respectively, with negative $\theta$-anomalies on the tropopause. The positive PV anomaly associated with the composite TPV approached 4 PVU above the lowered tropopause, peaking at the level of the composite background tropopause. Analysis of the tendency in anomalous PV due to diabatic processes demonstrated that this positive PV anomaly was enhanced primarily by radiative processes (anomalously strong cooling at the tropopause level and anomalously weak cooling above) with a weaker compensation, at the tropopause level, from latent heating. Cavallo and Hakim (2013) further showed, using idealised simulations, that TPV intensification is mainly due to anomalous longwave radiative cooling associated with the tropopause depression and displacement of the very low stratospheric specific humidity to much lower altitudes. The main climatological genesis region of TPVs is the Canadian archipelago and northern Baffin bay region, although genesis also occurs along and north of the Eurasian and North American coastline with a secondary maxima over the Kara sea (see Fig. 1 of Cavallo et al. (2009)). Despite the documented existence of some very long-lived cyclonic TPVs (Hakim and Canavan, 2005) suggesting long tracks, the climatological lysis regions lie immediately downstream of the genesis regions.

While forecast skill in the Arctic is comparable to that in the Northern hemisphere mid-latitudes, despite increased analysis uncertainty (Jung and Matsueda, 2016), forecast skill is poorer for Arctic cyclones than for mid-latitude cyclones. From analysis of ten "extraordinary" (large and intense) Arctic cyclones represented by ensemble forecasts from five operational centres, Yamagami et al. (2018b) found average location errors of about $470 \mathrm{~km}$ and central MSLP errors of 6-11 hPa for lead times of 2.5-4.5 days before maturity. The "Great Arctic cyclone of 2012" was notable for its exceptionally long lifetime (12 days) as well as intensity (minimum central MSLP of $966 \mathrm{hPa}$ ) (Simmonds and Rudeva, 2012). While the rapid intensification of this cyclone arose from lower-tropospheric baroclinicity (inferred from the Eady growth rate), a co-located TPV was important for the development of the surface cyclone. Analysis of the predictability of this cyclone revealed that this was increased by accurate prediction of upper-level, particularly temperature, features (Yamagami et al., 2018a). This cyclone formed due to the merging of both the upper-level warm cores (diagnosed as $250 \mathrm{hPa}$ temperature anomalies) and surface cyclones of a mid-latitude and Arctic cyclone, and the timing and southwards movement of a "polar vortex" (diagnosed using $300 \mathrm{hPa}$ geopotential height minimum) was crucial to the position and development of the merged cyclone; Yamagami et al. (2018a) noted that their definition of a polar vortex was similar to the TPVs defined by Cavallo et al. (2010). The geopotential height minimum on a pressure surface is associated with positive vorticity through geostrophic balance. The "warm cores" defined on pressure surfaces are positive $\theta$-anomalies and, as explained above, arise above TPVs because $\theta$-surfaces must dip downwards above the positive PV anomaly. Consistent with the findings of Yamagami et al. (2018a), Yamazaki et al. (2015) found that assimilation of additional radiosonde observations was crucial for accurate forecasts of this cyclone. Tao et al. (2017) also found that a polar vortex was important for the intensification of this cyclone, specifically through its role in intensifying the upper-tropospheric jet. A similarly exceptional Arctic cyclone developed in 2016, lasting for more than one month. This cyclone was maintained through multiple merging of cyclones and their associated warm-cored polar vortices (i.e., TPVs) (Yamagami et al., 2017).

It is not surprising that TPVs can have an important role in the formation and intensification of Arctic cyclones. Pre-existing upper-level PV anomalies, such as associated with upper-level troughs or smaller-scale jet steaks, have long been recognised 
as being able to initiate baroclinic growth in the mid-latitudes if they move over a low-level baroclinic zone (as classically described in Sect. 6e of Hoskins et al. (1985)). Petterssen and Smebye (1971) introduced the term "type B cyclogenesis" to describe the baroclinic mechanism of growth dominated by a finite amplitude upper-level precursor disturbance. This contrasts with "type A cyclogenesis" where a low level wave develops on a baroclinic zone, initially without much upper-level disturbance (although one may later develop). Deveson et al. (2002) later extended this classification scheme to include type $\mathrm{C}$ cyclogenesis, characterised by strong mid-level latent heat release. Considering nearly 700 cyclones in the North Atlantic region, Gray and Dacre (2006) diagnosed roughly equal numbers type A, B and C cyclones (30, 38 and $32 \%$ respectively) with type B cyclogenesis dominating for the Gulf Stream region off the East Coast USA. While the importance of upperlevel PV anomalies for cyclone development, as well as their almost ubiquitous existence as part of the three-dimensional mature cyclone structure (e.g. Čampa and Wernli, 2012), is well established in the mid-latitudes, evidence of the importance of pre-existing TPVs for Arctic cyclone development is more limited. Simmonds and Rudeva (2014) found that, at their time of maximum intensity, all but 6 of a set of 60 Arctic cyclones (the 5 most intense in each calendar month over a 30 year period) had a significant cyclonic feature in 300-hPa geopotential height within $555 \mathrm{~km}$; however, these upper-level cyclonic features were not explicitly linked to pre-existing TPVs. Tanaka et al. (2012) diagnosed an untilted structure with a vortex tube extending from the surface to the lower stratosphere at the mature stage of three Arctic cyclone case studies. Pre-existing TPVs have been associated with a few exceptionally large, intense and long-lived Arctic cyclones in case studies (Yamagami et al., 2018a, 2017), but their association more generally with typical cyclones is not known. In this study we explore this research gap by determining how often pre-existing TPVs are important for Arctic cyclone development over 40 extended summers (May-September). The following research questions are addressed:

- What are the statistical characteristics of Arctic cyclone tracks and how do they compare to those of TPVs?

- What is the role of TPVs in the initiation and intensification of Arctic cyclones?

- How is the structure of Arctic cyclones modified by interaction with TPVs?

The paper continues as follows. The methods are described in Sect. 2, beginning with the identification and feature tracking of Arctic cyclones and TPVs in 40 extended summers of ECMWF fifth generation (ERA5) reanalysis data (Hersbach et al., 2020) and then defining the method for matching Arctic cyclone and TPV features. Section 3 begins with quantification of the climatological characteristics of Arctic cyclone and TPV tracks before moving on to the spatial co-location of these features at the times of Arctic cyclone genesis, maximum growth rate and maximum intensity. Spatial composites are contrasted for two sets of Arctic cyclones, namely matched or unmatched with TPVs, at the time of maximum intensity, the earlier time of maximum growth rate and for two days before that. The composite structures are used to deduce the influences of TPVs on Arctic cyclone development and compared with those of mid-latitude cyclones. Section 4 contains the conclusions. 


\section{Methodology}

\subsection{Identification and feature tracking}

Arctic cyclones and TPVs are identified in the ERA5 dataset, the highest resolution reanalysis dataset available from ECMWF. Hourly data are available at a horizontal resolution of TL639 (approximately $30 \mathrm{~km}$ transform grid) with 137 terrain-following hybrid-pressure levels up to $80 \mathrm{~km}$. ERA5 was produced using cycle Cy41r2 of ECMWF's integrated forecast system which was operational from 8 March to 21 November 2016. Three-hourly data on a N320 Gaussian grid (approximate meridional grid spacing of $0.281^{\circ}$ ) was used for the extended summer season, May-September inclusive, from 1979-2018.

Arctic cyclones and TPVs were identified using maxima of $\xi_{850}$, and minima of $\theta$ on the dynamic tropopause (defined as the surface with PV of $2 \mathrm{PVU}), \theta_{2 \mathrm{PVU}}$, respectively. A minimum track distance of $1000 \mathrm{~km}$ was imposed to focus on mobile systems. The TRACK algorithm (version 1.5.2) was used to track the identified features (Hodges, 1994, 1995, 1999). For tracking, mid-latitude cyclones are typically identified using either $\xi_{850}$ or MSLP (e.g. Neu et al. (2013)). The $850 \mathrm{hPa}$ level is used for $\xi$ to avoid strong influences from boundary layer processes and orography. As described in Sect. 1, both fields were used in a recent comparison of Arctic cyclones tracked using different global reanalyses that used the same tracking algorithm as used in this study (Vessey et al., 2020). The authors found that more Arctic cyclones were identified using $\xi_{850}$ than using MSLP due to the identification of smaller-scale systems using $\xi_{850}$. In the summer about $50 \%$ more cyclones were identified using $\xi_{850}$, whereas in the winter the values nearly doubled. Note though that these findings are likely to be somewhat dependent on the spatial filtering before tracking and the post-tracking filtering. Far fewer studies have tracked TPVs and our identification of these systems using $\theta_{2 \mathrm{PVU}}$ minima follows that of Cavallo et al. (2010).

Spatial filtering of the fields is required prior to feature identification and tracking to remove both the planetary-scale background flow and smaller mesoscale features and so focus on the scales of interest. Spectral filtering was applied to retain features with wavenumbers in the range T5-T63 (where TN refers to triangular truncation of the spherical harmonics) for both fields to yield anomalies. Data were then projected to a $200 \times 200$ grid on a polar projection for feature identification before the feature points were converted back to the sphere for tracking. Note that because the atmospheric evolution is close to adiabatic and frictionless, to a good approximation $\theta$ is advected conservatively on the 2 PVU surface and therefore no maxima or minima can be created in $\theta$ that are not in the initial conditions. Hence we track minima in $\theta$-anomalies, which can be generated by equatorward displacement of air with low $\theta$ values, rather than minima in the $\theta$ field itself. In contrast, by definition $\xi$ is already an anomaly relative to the planetary vorticity distribution and so the weak background field does not need to be removed; however, this removal is done for consistency. Preliminary case study analysis in which different spectral filtering ranges were compared (not shown) showed the chosen filtering retained features of interest while removing smaller mesoscale features, such as fronts, and smaller TPV features. For tracking several thresholds were employed. For initial identification for the tracking Arctic cyclones were identified in $\xi_{850}$ using a threshold of $10^{-5} \mathrm{~s}^{-1}$ for the maxima and TPVs were identified in $\theta_{2 \mathrm{PVU}}$ using a threshold of less than $-1 \mathrm{~K}$ for the minima. Following the tracking, tracks were retained if they existed for more than 1 day (8 time steps) and the track distance exceeded $1000 \mathrm{~km}$. 


\subsection{Post-processing of tracked features}

Tracks for both TPV and Arctic cyclones were partitioned into those that formed within the Arctic region (defined here as North of $65^{\circ} \mathrm{N}$, following Vessey et al. (2020)) and those that formed further south and moved into the Arctic. To determine the role of TPVs in the initiation and intensification of Arctic cyclones, Arctic cyclones were matched to TPVs at the times of genesis, maximum growth rate and maximum intensity of the Arctic cyclones. The matching was performed for horizontal separation geodesic distances (matching radii) of between $1^{\circ}$ and $5^{\circ}$. The genesis time of features is defined as the first time at which they are identified (above threshold intensity). The time of maximum growth rate of cyclones is defined at the start of the three-hour period over which the filtered $\xi_{850}$ has the maximum increase along the track. The time of cyclone maximum intensity is defined by the filtered $\xi_{850}$ maximum along the track.

Two subsets of Arctic cyclones are defined: those that are matched and those that are unmatched to a TPV during their intensification period (termed matched and unmatched cyclones). Matched cyclones are defined as cyclones that are within $2^{\circ}$ of an Arctic origin TPV at the time of their maximum intensity and within $5^{\circ}$ of an Arctic origin TPV at the time of their maximum growth rate. Unmatched cyclones are defined as cyclones that are further than $5^{\circ}$ from a TPV at both their time of their maximum growth rate and time of their maximum intensity. These feature separation thresholds were chosen assuming that the Arctic cyclones evolved from a more tilted vertical structure, conducive to baroclinic growth, to a more vertical structure at maturity (maximum intensity). Both matched and unmatched cyclones must also exist for at least two days prior to their maximum growth rate and achieve their maximum intensity in the Arctic. This enables consistent investigation of the structure of Arctic cyclones at one and two days prior to maximum growth rate. The Rossby deformation radius in the Arctic is estimated to be at most $500 \mathrm{~km}$ (for a tropopause at $7 \mathrm{~km}$ and Coriolis parameter of about $1.5 \times 10^{-4} \mathrm{~s}^{-1}$ ) and so TPVs were considered to be potentially capable of contributing to the baroclinic intensification of an Arctic cyclone if they were within $5^{\circ}$. Note though that the TPVs are not constrained to be located such that the direction of the tilt between a TPV and the associated surface Arctic cyclone is conducive to baroclinic growth (i.e. rearwards relative to the movement of the cyclone). The matching radius was reduced from $5^{\circ}$ at maximum growth rate to $2^{\circ}$ at maximum intensity to take into account the expected more vertical structure at this stage.

Composite matched and unmatched cyclones were generated using the 200 most intense cyclones (defined by the filtered $\xi_{850}$ at the time of maximum intensity) from the matched and unmatched sets, respectively. The composites were generated by first setting up a rectangular grid centred on the equator. This grid was then moved to the centre of the matched or unmatched Arctic cyclone and orientated relative to the direction of cyclone movement so that all cyclones are orientated in the same direction relative to their motion. Data were then sampled to the grid and finally averaged over the cyclones. The number of cyclones for compositing was chosen as a compromise between choosing too few cyclones, such that the composite fields plotted may not be representative, and too many, such that cyclone features may be smeared out by large differences between the most and least intense systems.

The tilt structure of the composite cyclones, defined by the vertical structure of $\xi$ maxima on selected pressure levels, was calculated based on the method used in Bengtsson et al. (2009). The tilt for each of the 200 matched (or unmatched) cyclones 
was calculated recursively by identifying the maxima at each level using the maxima at the previous level as a starting point for the search. The search was performed starting at $900 \mathrm{hPa}$ (the bottom of the profile) in the filtered data and continuing until $50 \mathrm{hPa}$, using a search radius of $3^{\circ}$ (though similar results were obtained using a search radius of $5^{\circ}$ (not shown)). A steepest ascent and B-spline interpolation method was used to determine the maxima. These maxima were then projected onto the cyclone motion direction so that the tilt was relative to this direction (in spherical geometry) and the position relative to the $850 \mathrm{hPa}$ centre (the level at which the cyclone motion direction is defined) determined as a geodesic angle. The tilts were then adjusted to be relative to the $900 \mathrm{hPa}$ location. Finally, the radii were averaged at each level over the set of matched (or unmatched) cyclones relative to the time of maximum growth rate to yield the three-dimensional composite structure. Note that occasionally a sufficiently close $\xi$ maximum could not be found at a level; this becomes more likely at higher levels. The composite tilt structure was calculated from two days before to two days after the time of maximum growth rate. While each cyclone must exist from at least two days before the time of its maximum growth rate due to the criteria for selection of the matched and unmatched cyclones described above, there is no requirement for their existence after this time. Hence, a small number of cyclones do not have identified tracks for the full two days after their time of maximum intensity.

\section{Results}

\subsection{Climatological characteristics of TPVs and Arctic cyclones}

The genesis density of tracked Arctic cyclones and TPVs is shown in Fig. 1 (top and bottom row, respectively) both for all systems and split into systems with genesis within and outside the Arctic. Considering first the Arctic cyclones, there are clear enhanced regions of genesis along several coastlines around the Arctic. The strongest genesis region lies along the east coast of Greenland. Arctic cyclones with genesis outside of the Arctic have preferred genesis regions over the Canadian Rockies and southern Scandinavia (Fig. 1(c)). These preferred genesis regions are consistent with previous studies performed using different reanalyses (e.g., Vessey et al., 2020). The genesis locations of TPVs are less localised than those of Arctic cyclones (Fig. 1(d)). However, there are still a few notable areas of enhanced genesis density. The strongest of these areas is located over northern Canada and the Canadian Arctic Archipelago (as also found by Cavallo et al. (2009)). Other density maxima are located over the North Pole and northern Siberia. It is rare for TPVs to have genesis regions outside of the Arctic (Fig. 1(f)), although they can migrate out of the Arctic consistent with the negative $\theta$-anomalies (on the tropopause) tending to be associated with equatorward displacement.

The track density of tracked Arctic cyclones and TPVs is shown in Fig. 2 organised in the same way as for genesis density. The track density plots are smoother than those of genesis density and the track density of Arctic cyclones is much more evenly spread across the Arctic Ocean than the genesis density. So, although the Arctic cyclones tend to be preferentially first identified near the coastlines, many subsequently track towards the North Pole. Nevertheless, there is enhanced track density over northern Russia, which is the main area influenced by Arctic cyclones with genesis regions both within and outside the Arctic. The only area of the Arctic where Arctic cyclones are not observed is across the centre of Greenland; this gap is a consequence of the high altitude of the Greenland plateau which means that it lies above the $850 \mathrm{hPa}$ surface used to identify 
https://doi.org/10.5194/wcd-2021-30

Preprint. Discussion started: 7 June 2021

(c) Author(s) 2021. CC BY 4.0 License.

Weather and

Climate Dynamics

(c) (i)

(a)

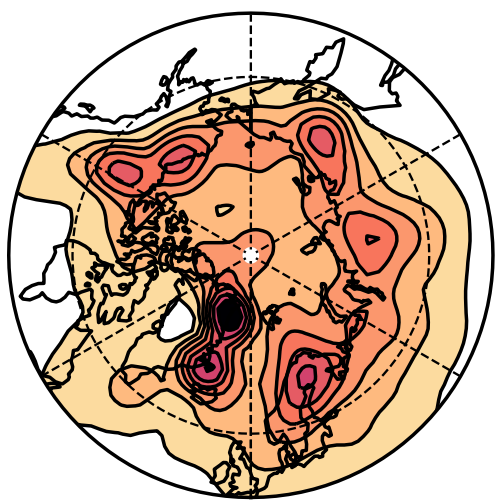

(d)
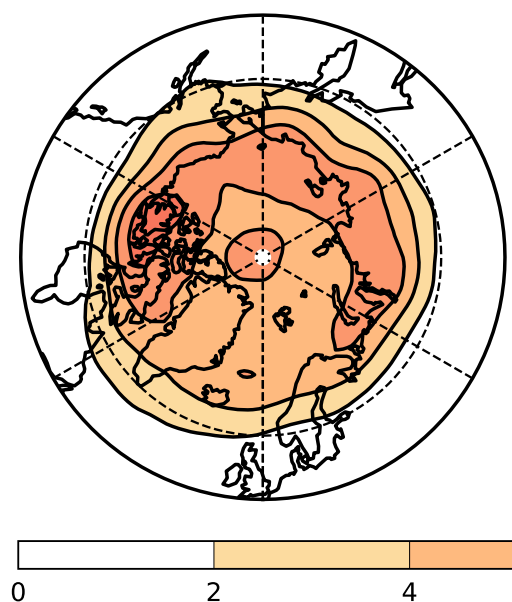

(b)

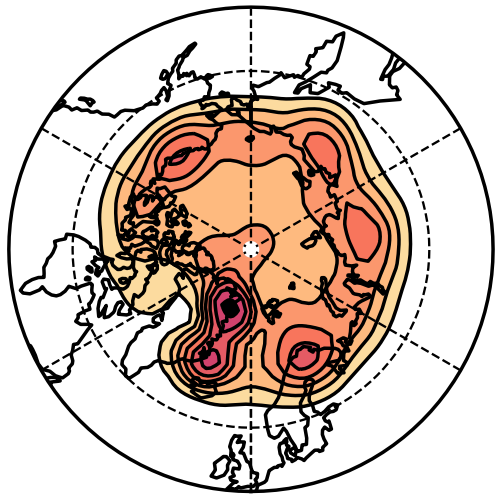

(e)
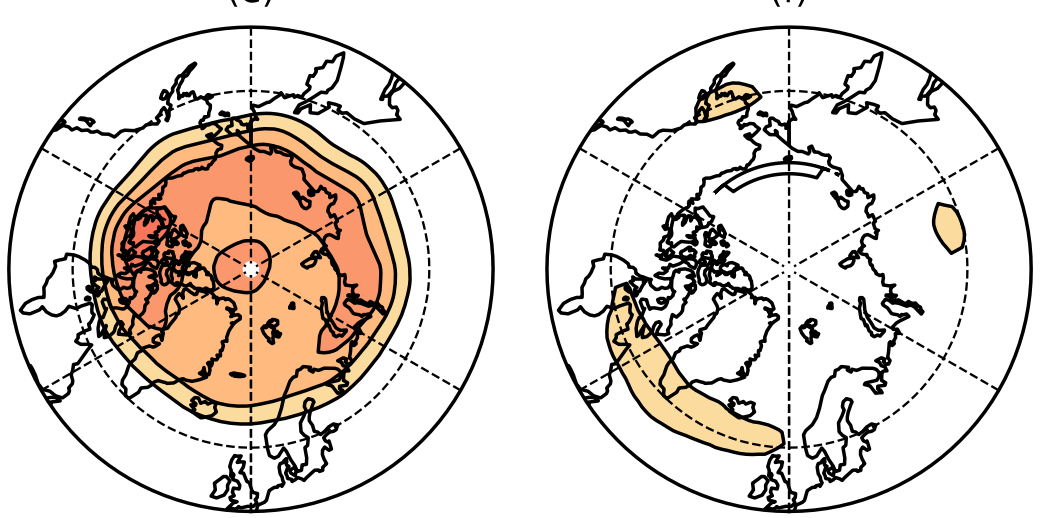

(f)

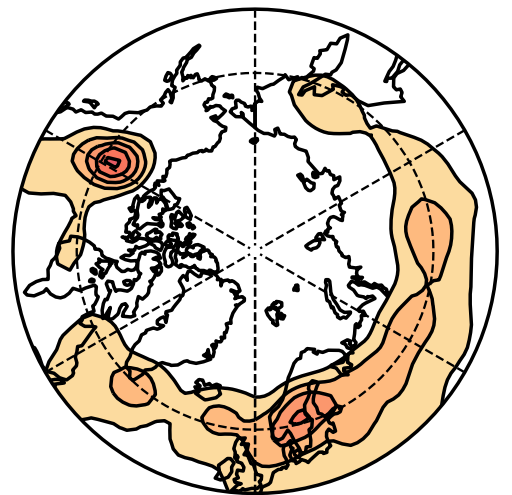

14

Figure 1. Genesis density of Arctic cyclones for a) all cases, b) Arctic genesis only and c) non-Arctic genesis. Genesis density of TPVs for d) all cases, e) Arctic genesis only and f) non-Arctic genesis. Data spans the extended summer season between 1979 and 2018 . Units are number per unit area per season where the unit area is equivalent to a five degree spherical cap $\left(\sim 10^{6} \mathrm{~km}^{2}\right)$. The total number of tracks is a) 12155 , b) 6822 , c) 5333 , d) 8339 , e) 6288 , and f) 2051 . Maps are orientated with $0^{\circ}$ longitude at the bottom.

cyclones leading to disruption of the tracking. Unlike for Arctic cyclones, the track density plots for the TPVs are somewhat similar to those for genesis density. This result is consistent with the finding of Cavallo et al. (2009) that the TPV lysis regions are immediately downstream of the genesis regions. Figure 2(d) also shows a density maximum over the Canadian Arctic Archipelago, though slightly further east than for genesis density. The rest of the tracks are found along the Arctic coastlines, at an approximate latitude of $70^{\circ} \mathrm{N}$. Notably, there is a relatively lower track density in the central Arctic Ocean. In particular, very few TPVs with genesis outside the Arctic make it into the central Arctic region.

The distributions of lifetimes and intensities of the tracked Arctic cyclones and TPVs are shown in Fig. 3 (left and right panels, respectively); note that in all panels the means of the two distributions plotted are significantly different at the $95 \%$ level 
https://doi.org/10.5194/wcd-2021-30

Preprint. Discussion started: 7 June 2021

(c) Author(s) 2021. CC BY 4.0 License.

Weather and

Climate Dynamics

(c) (i)

(a)

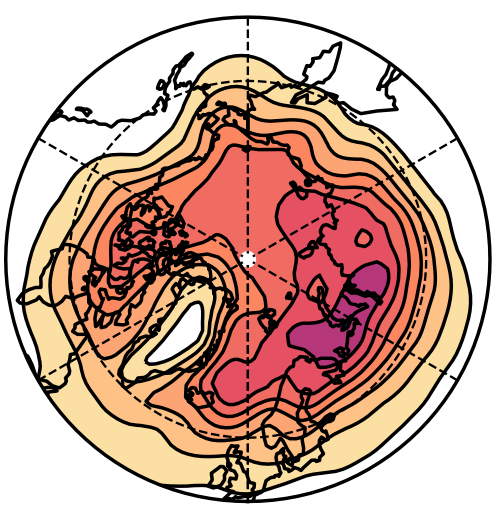

(d)

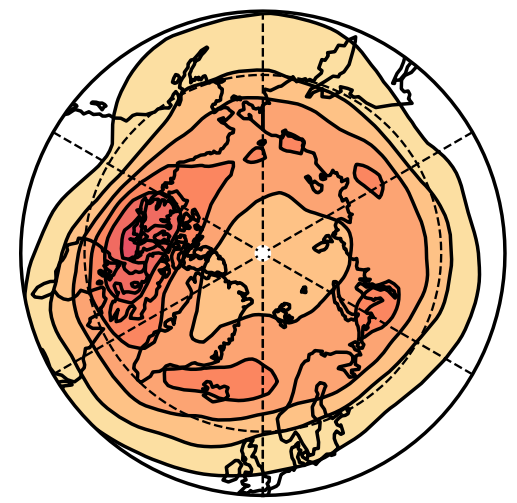

(b)

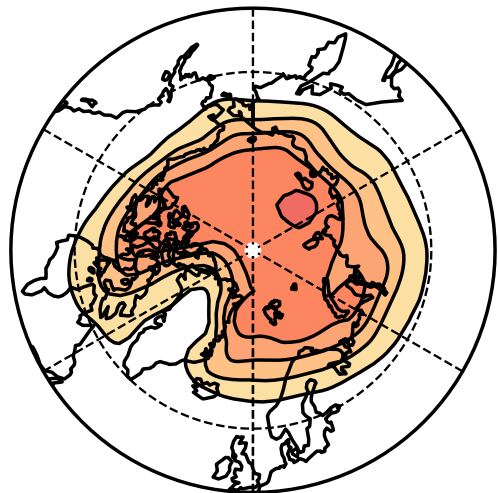

(e)

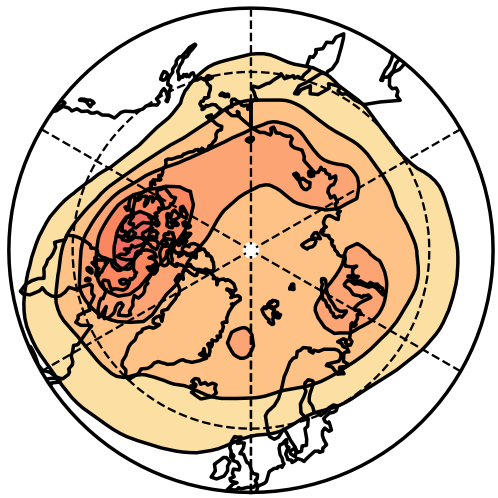

(c)

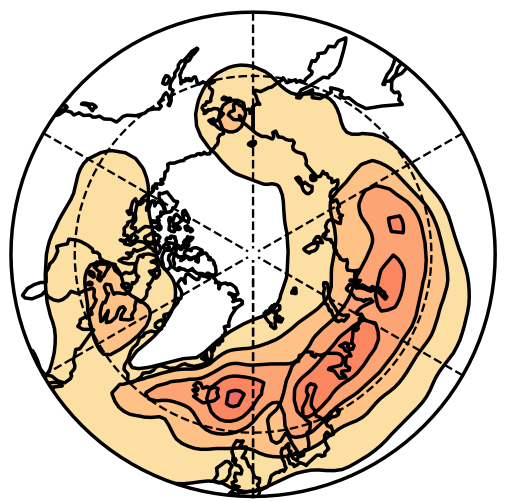

(f)

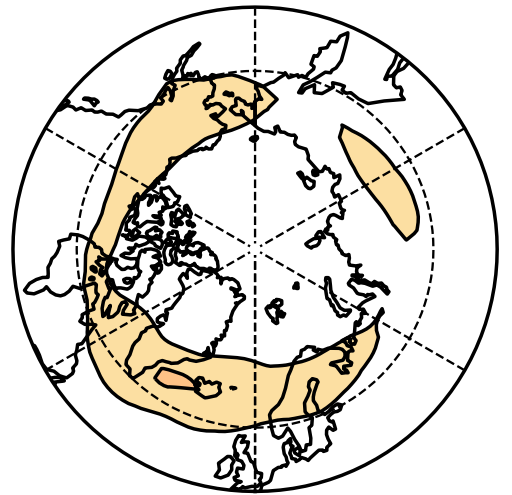

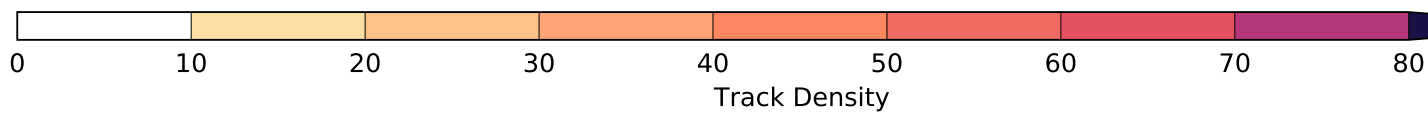

Figure 2. As for Fig. 1 but for feature track density. (a, b, c) for Arctic cyclones and (d, e, f) for TPVs.

according to a two-sided Welch's t-test (without assuming equal variance). Lifetime distributions are compared for the Arctic cyclones and TPVs (Fig. 3(a)) and the Arctic and non-Arctic genesis cyclones (Fig. 3(c)) and TPVs (Fig. 3(e)), respectively. The modal lifetime of Arctic cyclones of 2-3 days exceeds that of TPVs (1-2 days), but the number of Arctic cyclones drops off more steeply with lifetime than for TPVs, and the Arctic cyclone lifetime distribution has a shorter and sparser tail than that of TPVs (Fig. 3(a)). Consequently the mean TPV lifetime is slightly longer than that of Arctic cyclones (5.0 compared to 4.4 days). Some exceptionally long-lived TPVs exist with the maximum lifetime found being 43 days. It is possible that exceptionally long lifetimes could result from the tracking algorithm erroneously connecting tracks associated with two different TPVs.

However, more detailed examination of some of these tracks suggests that they are reliable; Hakim and Canavan (2005) also found that TPV lifetimes could exceed one month. We speculate that some of these long-lived TPVs are the remnants of the tropospheric polar vortex which becomes much smaller moving into summer and typically breaks into a few smaller PV features at some point. These TPVs are long lived because they are coherent stronger vortices which resist the relatively weak 
https://doi.org/10.5194/wcd-2021-30

Preprint. Discussion started: 7 June 2021

(c) Author(s) 2021. CC BY 4.0 License.

Weather and

Climate Dynamics

Discussions

L

large-scale strain and also because longwave cooling maintains the positive PV anomaly where the tropopause is lower. Arctic cyclones are more likely to be long-lived if they track into the Arctic region than if they have their genesis there (Fig. 3(c), mean lifetimes 4.9 and 3.9 days, respectively). Conversely, TPVs are likely to be longer-lived if they have Arctic, rather than non-Arctic genesis (Fig. 3(e), mean lifetimes 5.2 and 4.4 days, respectively).

Distributions of intensity characteristics are shown in Fig. 3(b,d,f) partitioned into systems with Arctic and non-Arctic genesis. Arctic cyclones with non-Arctic genesis tend be more intense defined either by their maximum $\xi$ or their minimum MSLP (at the time of maximum $\xi$ ). Conversely, TPVs tend to be more intense, diagnosed from their maximum $\theta_{2 \mathrm{PVU}}$ anomaly, when their genesis region is in the Arctic (noting though that the number of non-Arctic genesis TPVs is only about a third of that of Arctic genesis TPVs).

A time series of the annual count of tracked TPVs and Arctic cyclones (with lifetimes of at least 1 day) for each year between 1979 and 2018 is shown in Fig. 4, for all systems and split between those of Arctic and non-Arctic genesis. Arctic cyclones are more numerous than TPVs every year, with average annual counts over the 40-year study period of 304 and 208 respectively. TPVs are more likely than Arctic cyclones to have Arctic genesis: $75 \%$ of TPVs have Arctic genesis compared to $55 \%$ for Arctic cyclones. The number of Arctic cyclones (and their Arctic genesis proportion) found here using ERA5 can be compared with the equivalent values found using other reanalyses. Vessey et al. (2020) found an average of 97 (range 96.2-98.3) Arctic cyclones with lifetimes exceeding two days occurred each June-August summer season in the ERA-Interim, JRS-55, MERRA-2 and NCEP-CFSR reanalyses (1980-2017) with an average of 47.4\% (range 47.0-47.8 \%) having Arctic genesis. The definitions of the Arctic region and the tracking algorithm and tracked field used in Vessey et al. (2020) are the same as used here; however, in Vessey et al. (2020) T5-T42 spectral filtering was applied to the $\xi_{850}$ field whereas here T5T63 filtering was used, so retaining some smaller-scale systems. Even adjusting for the longer summer season considered here (five instead of three months), substantially more Arctic cyclones are found although the percentage that have Arctic genesis is similar. The longer minimum lifetime allowed by Vessey et al. (2020) is not the main reason for the disparity as, using the same minimum lifetime as Vessey et al. (2020), we obtain an average of 266 Arctic cyclones. Hence, the higher resolution of ERA5 (compared to the reanalyses used by Vessey et al. (2020)) and allowed smaller-scale of tracked cyclones yields the higher cyclone counts, indicating the sensitivity of the results to these factors.

The time series of TPVs and Arctic cyclones are significantly correlated at the $95 \%$ level (Pearson correlation) for both nonArctic and Arctic genesis (correlation coefficients of 0.366 and 0.324, respectively, with corresponding two-tailed p-values of 0.020 and 0.042 , respectively), but this significance drops to to $10 \%$ (correlation coefficient of 0.299 , p-value 0.061 ) when correlating all Arctic cyclones with all TPVs. The time series do not appear to have notable trends from visual inspection of Fig. 4 and the significant correlations are retained after detrending by linear model fitting (not shown). The number of Arctic cyclones is also correlated with the North Atlantic Oscillation (NAO) (index time series downloaded from National Weather Service Climate Prediction Center (2021)) such that more cyclones occur in the positive phase. The correlations are significant at the $95 \%$ level taking all cyclones and those with non-Arctic genesis only (correlation coefficients of 0.453 and 0.374 and p-values 0.003 and 0.017 , respectively). This significance drops to the $90 \%$ level for correlation with Arctic genesis cyclones (correlation coefficient of 0.297 , p-value 0.062 ). This correlation is consistent with more cyclones tracking northeastwards into 
https://doi.org/10.5194/wcd-2021-30

Preprint. Discussion started: 7 June 2021

(c) Author(s) 2021. CC BY 4.0 License.

Weather and

Climate Dynamics

(c) (i)

Discussions

L

the Arctic region from the North Atlantic sector during positive NAO when the jet stream is strong. There is no significant

315 correlation between the number of TPVs and the NAO index. The correlation between the time series of Arctic cyclones and TPVs is consistent with TPVs having a role in the initiation and/or intensification of Arctic cyclones, as investigated in the following section. 
(a)

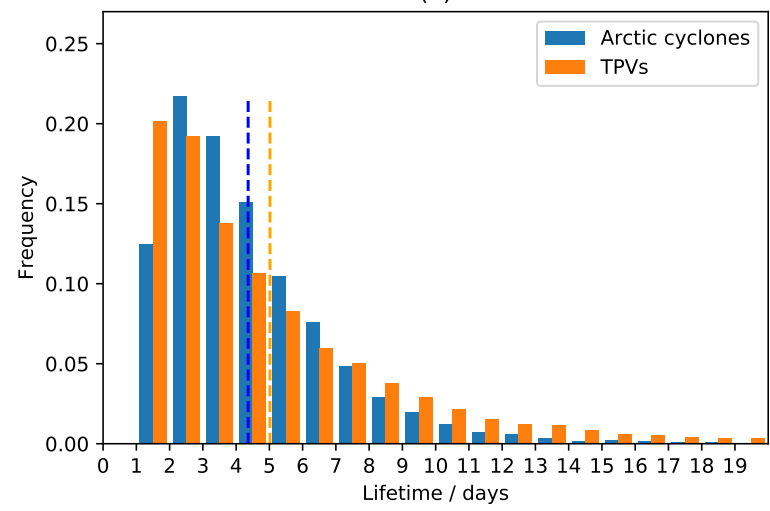

(c)

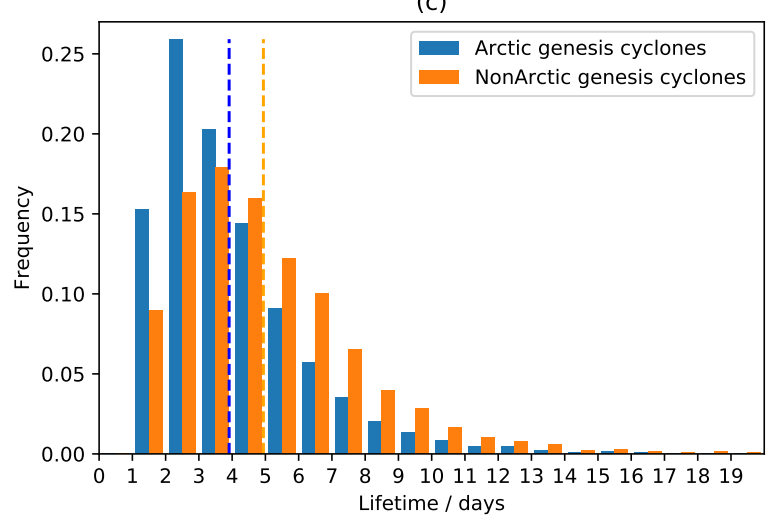

(e)

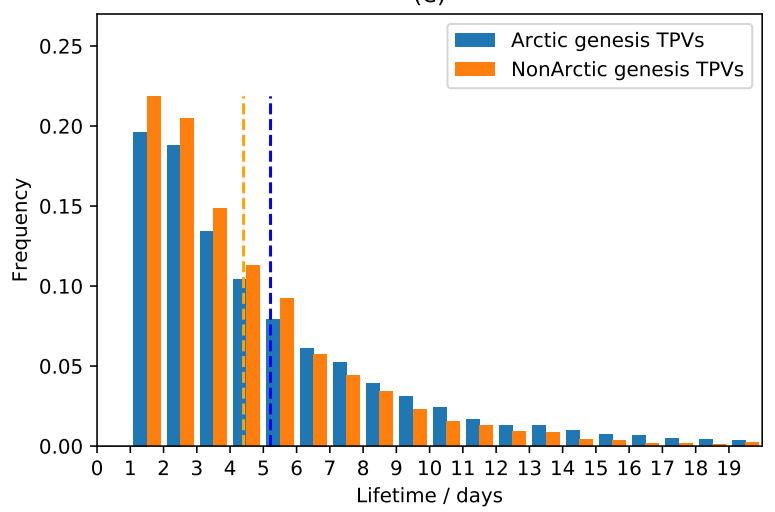

(b)

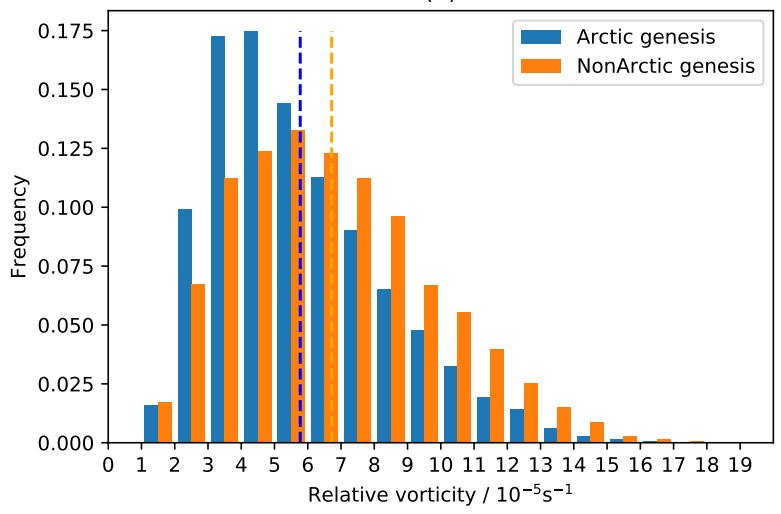

(d)

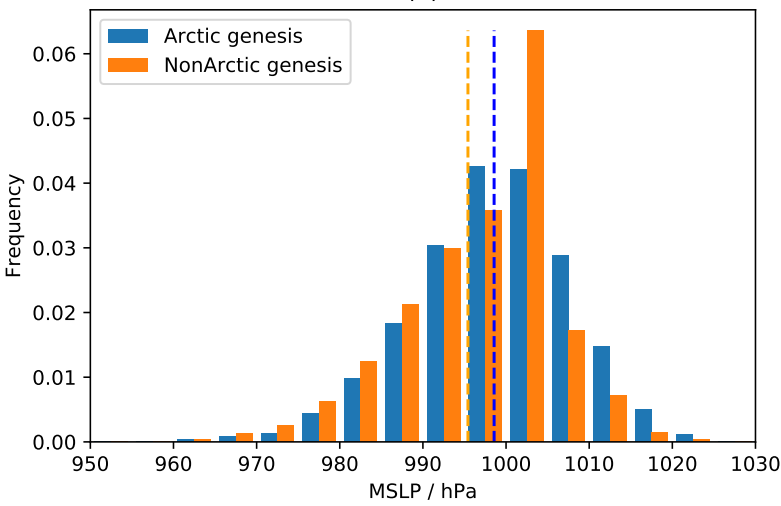

(f)

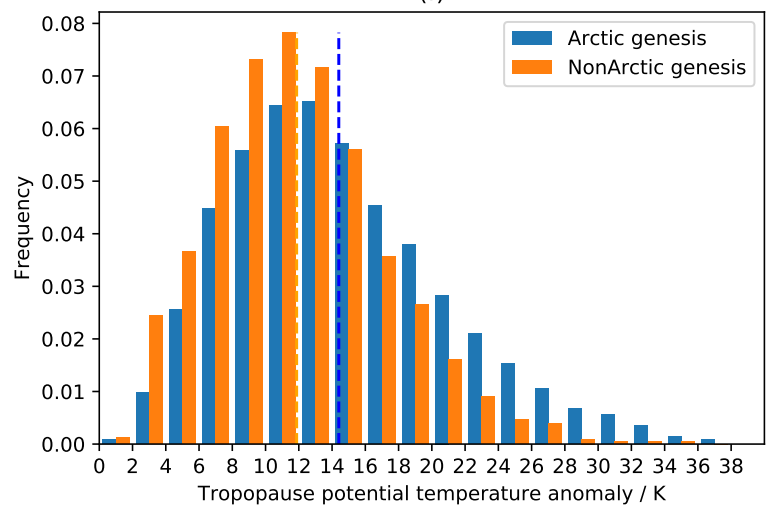

Figure 3. Normalised frequency plots of Arctic cyclone and TPV characteristics: (a,c,e) lifetimes of Arctic cyclones and TPVs out to 20 days (maximum lifetimes are 27 and 43 days respectively) with (a) for all Arctic cyclones and TPV systems, (c) for Arctic cyclones partitioned into Arctic and non-Arctic genesis, and (e) for TPVs partitioned into Arctic and non-Arctic genesis; and (b,d,f) intensity of systems partitioned into Arctic and non-Arctic genesis with (b) maximum filtered $\xi_{850}$ of Arctic cyclones, (d) MSLP at the time of maximum $\xi_{850}$ of Arctic cyclones, and (f) maximum filtered $\theta^{\prime}$ on the 2-PVU surface. Mean values are indicated by vertical dashed lines in each panel. The minimum lifetime of tracked systems is 1 day. In (a,c,e) the first pair of bars presents the frequency of systems with 1 day $\leq$ lifetime $<2$ days, the second pair systems with 2 days $\leq$ lifetime $<3$ days etc. An analogous interpretation of the bars applies for the intensity plots. 
https://doi.org/10.5194/wcd-2021-30

Preprint. Discussion started: 7 June 2021

(c) Author(s) 2021. CC BY 4.0 License.

Weather and

Climate Dynamics

(c) (i)

Discussions

L

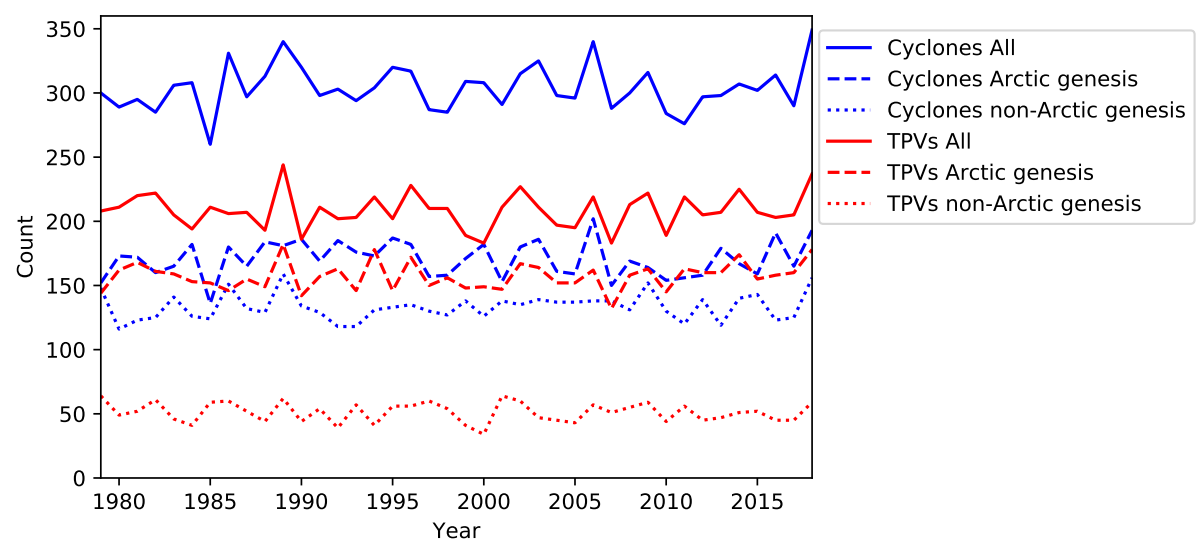

Figure 4. Time series of extended-summer counts of Arctic cyclones and TPVs with a lifetime of $\geq 1$ day 


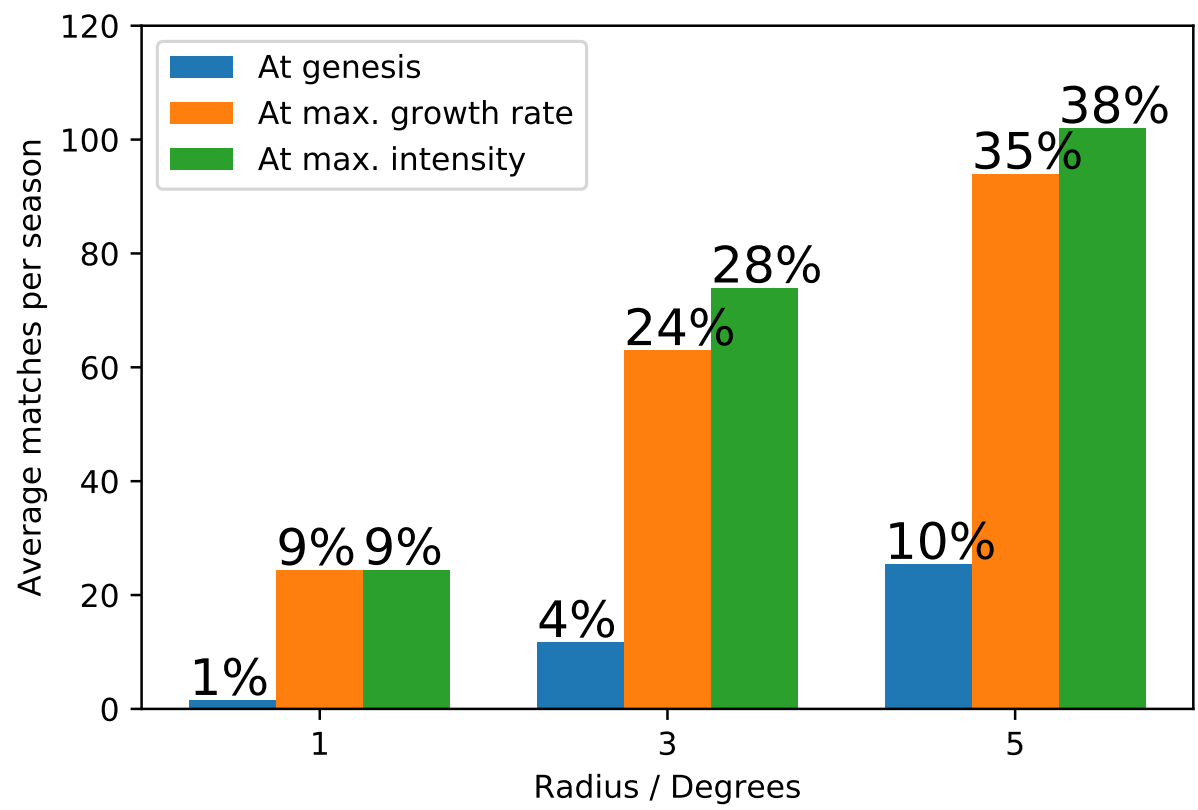

Figure 5. Matches between (all) Arctic cyclones with lifetimes $\geq 2$ days and Arctic genesis TPVs at times of genesis, maximum growth rate and maximum intensity of the Arctic cyclone for selected spatial overlap radii (note that 1 degree is equivalent to $111 \mathrm{~km}$ in great circle distance). Values above the bars are the percentage of Arctic cyclones that are matched.

\subsection{Spatial association between Arctic cyclones and TPVs}

The potential role of TPVs in the initiation and/or intensification of Arctic cyclones is explored by assessing the proximity between these systems at three times: the times of genesis, maximum growth rate and maximum intensity of the Arctic cyclones. Figure 5 shows these matches for TPVs occurring within 1, 3 and $5^{\circ}$ of the Arctic cyclones (termed the overlap radius and defined as the arc length of great circle (geodesic) centred on the Arctic cyclone). As expected, the number of matches increases with the overlap radius. While TPVs are near to some Arctic cyclones at their genesis time, TPVs are far more likely to be near to Arctic cyclones when the Arctic cyclones are intensifying rapidly. For a $5^{\circ}$ overlap radius $35 \%$ of Arctic cyclones are matched with a TPV at the time of maximum Arctic cyclone growth rate compared to only $10 \%$ at the time of Arctic cyclone genesis. This suggests that while TPVs can have a role in the intensification of Arctic cyclones, they are more likely to either co-develop with the Arctic cyclone (i.e. form as a consequence of the three-dimensional baroclinic development) or lead to rapid intensification of an already developing Arctic cyclone as they track into the vicinity of the surface cyclone. The percentage of cyclones matched with TPVs at the time of cyclone maximum intensity is similar to, or slightly larger than, at the time of maximum growth rate (for a given overlap radius), implying that the spatial association between the TPV is maintained until at least this time. Despite the potential importance of TPVs for Arctic cyclone intensification, the majority (about two thirds) of Arctic cyclones are not in close proximity to a TPV at the time of their maximum growth rate. 
https://doi.org/10.5194/wcd-2021-30

Preprint. Discussion started: 7 June 2021

(c) Author(s) 2021. CC BY 4.0 License.

Weather and

Climate Dynamics

Discussions

L

The geographical locations where the matching between TPVs and Arctic cyclones occurs is now determined. Maps of the genesis and track densities show large differences between the matched and unmatched cyclones (Fig. 6). The chosen criteria led to a reasonably similar number of cyclones (about 400) being identified as matched and unmatched, simplifying the interpretation of the comparison between the density maps. As for the full set of Arctic cyclones (Fig. 1(a)), large genesis densities of both the matched and unmatched cyclones are found in two localised maxima to the east side of Greenland with larger values in the more northern maxima. Northern Canada, including the Canadian Arctic Archipelago, is a more important genesis region for the matched cyclones. Conversely, the genesis regions over northern Russia and northern Scandinavia (the Arctic Frontal Zone) are relatively more important for the unmatched cyclones. An additional localised maximum over the North Pole also appears for the matched cyclones. As for the full set of Arctic cyclones, the track densities for the matched and unmatched cyclones are much smoother than the genesis densities. The differences between the track densities for the matched and unmatched cyclones can be interpreted using the track densities for the full set of Arctic cyclones and Arctic genesis TPVs shown in Fig. 2(a) and (e), respectively. As the track density for Arctic genesis TPVs is largest over the Canadian Arctic Archipelago so the track density of the matched Arctic cyclones is also largest there, with an extension westwards over the Arctic Ocean to the north of Canada (to the north of the enhanced genesis density in Fig. 6(a)). In contrast, for the unmatched Arctic cyclones, the track density is largest over the Barents and Kara Seas along the Russian coastline, consistent with these systems relying on the strongest section of the Arctic Frontal Zone where there is also a low-level jet (Day and Hodges, 2018).

Finally, Fig. 7 shows how the matching changes with month during the extended summer season. The constraint that the cyclones must exist for at least two days prior to their maximum growth rate time has been removed here to generate a larger dataset (of 1096 and 3094 matched and unmatched cyclones, respectively); otherwise the subsets are defined as in Sect. 2.2. While there is no strong monthly variability, there are indications of anti-correlation in the time series. There is a general tendency for the number of matched cyclones to decrease to a minimum in August before increasing again in September with a corresponding increase in the number of unmatched cyclones in late summer before decrease in September.

\subsection{Composite structure evolution of Arctic cyclones matched and unmatched with a TPV}

The interaction between TPVs and Arctic cyclones is now investigated by comparing the composite structures of the matched and unmatched cyclones (calculated as described in Sect. 2.2). The 200 most intense cyclones from the sets of matched and unmatched cyclones already defined were selected for compositing. The structural evolution of the matched and unmatched cyclones are shown in Figs. 8 and 9, respectively, at four times: two and one days prior to the time of maximum growth rate, maximum growth rate and maximum intensity. To aid the description of results in this section, the cyclone motion direction (indicated by the grey arrows in Figs. 8 and 9) is nominally considered eastwards. The left columns of each plot show the evolution in near surface fields ( $\theta_{900}^{\prime}$ and MSLP). The anomaly field $\theta^{\prime}$ (relative to the domain average), is plotted rather than the full $\theta$ field to allow for the seasonal temperature variation over the extended summer season considered. The evolution of the matched and unmatched composite cyclones are very similar in these fields. A warm sector develops on the southeast flank of the MSLP centre of the cyclone from a northeast-southwest temperature gradient two days prior to maximum growth rate. This reaches a peak $\theta^{\prime}$ and closest proximity to the centre of the cyclone at the time of maximum growth rate. The cold sector 
(a)

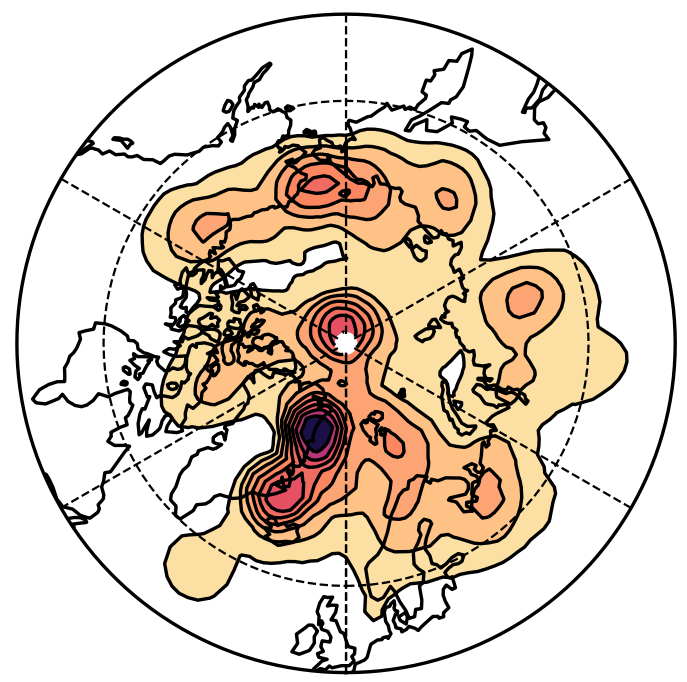

(c)

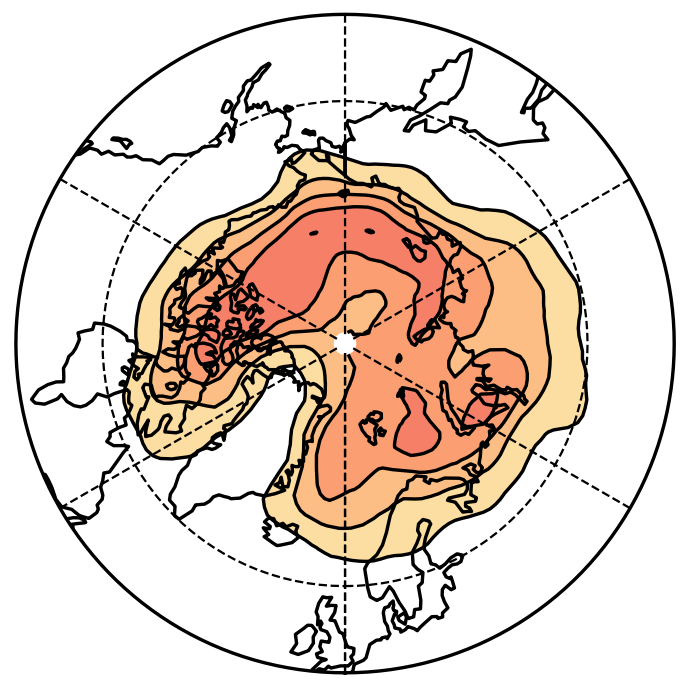

(b)

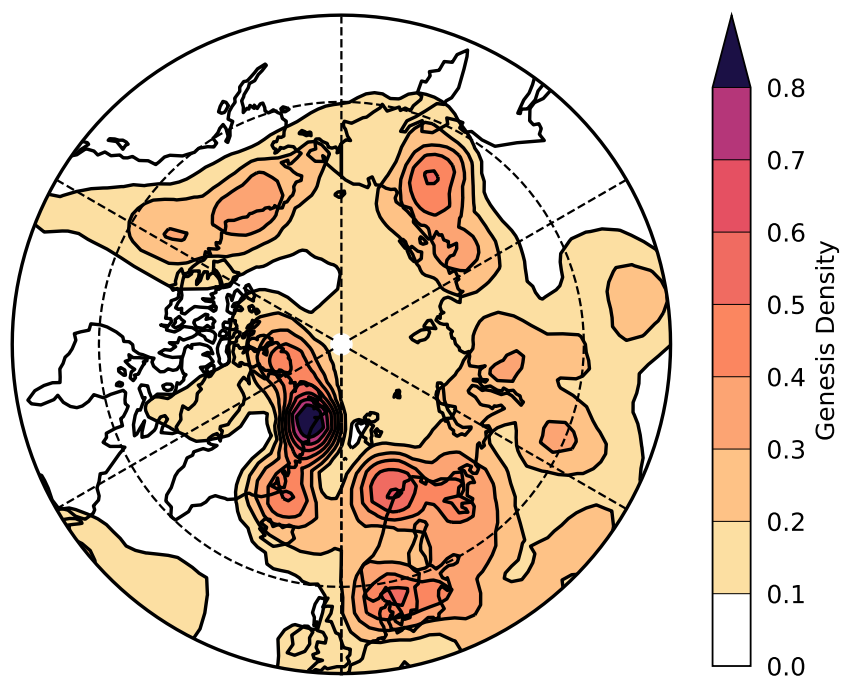

(d)

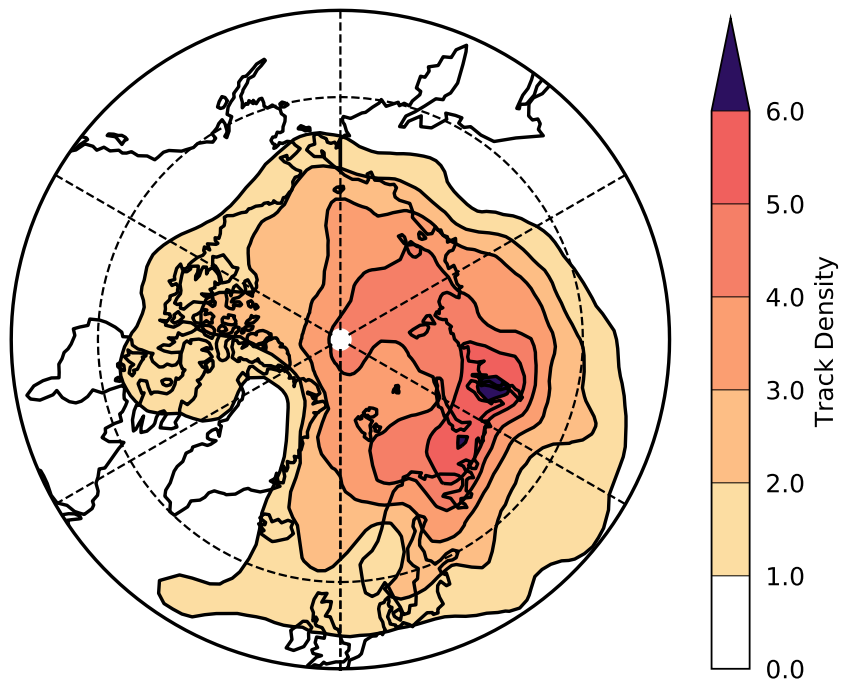

Figure 6. Genesis and track densities of Arctic cyclones matched and unmatched to a TPV. a) matched and b) unmatched cyclone genesis densities and c) matched and d) unmatched cyclone track densities during the extended summer season between 1979 and 2018. Units are number per unit area per season where the unit area is equivalent to a 5 degree spherical cap $\left(\sim 10^{6} \mathrm{~km}^{2}\right)$. The total number of tracks is 341 for the matched cyclones and 470 for the unmatched cyclones. Maps are orientated with $0^{\circ}$ longitude at the bottom.

gradually wraps around the cyclone centre to the northwest as the cyclone intensifies and the $\theta^{\prime}$ at the centre of the cyclone cools between the times of maximum growth rate and maximum intensity. In both composites the minimum MSLP decreases to approximately $990 \mathrm{hPa}$ as the cyclone evolves towards maximum $\xi$ intensity. However, the low-level $\theta$-wave and MSLP perturbation amplitude are slightly stronger in the unmatched cyclone composite. 


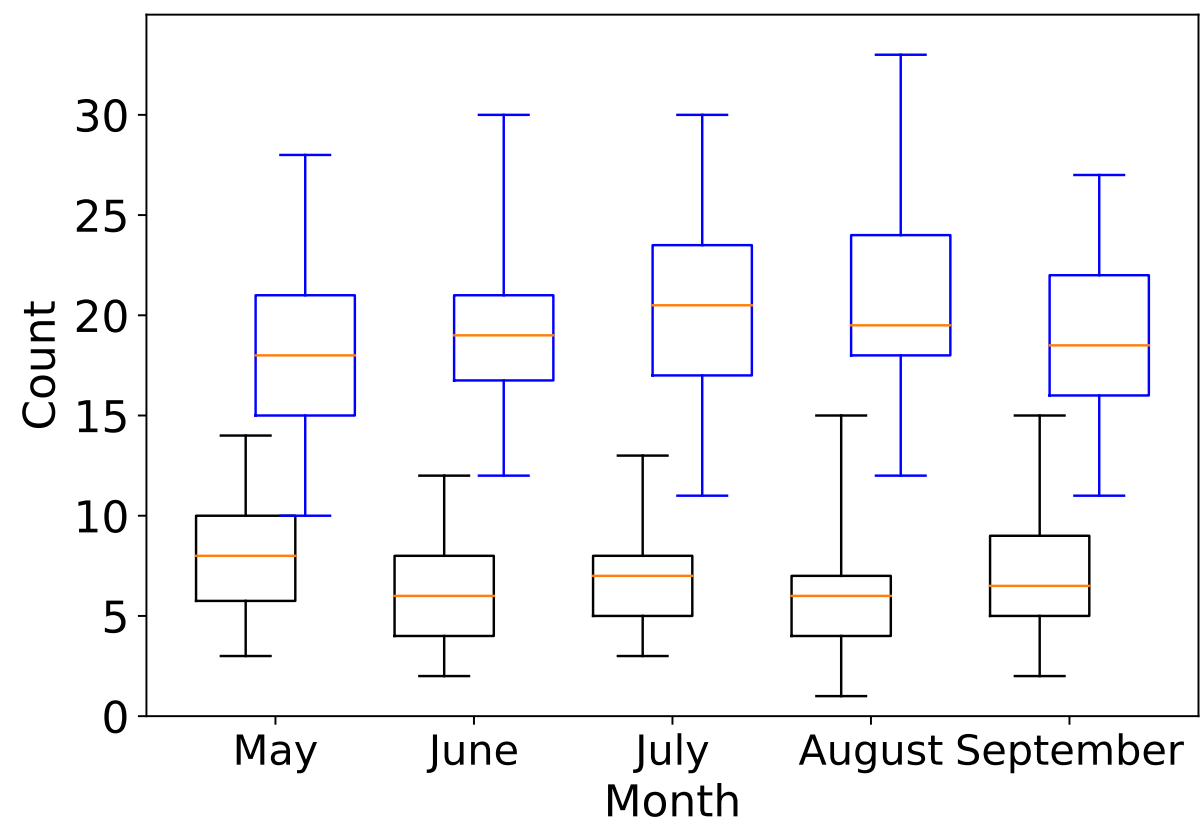

Figure 7. Box and whisker plot of the monthly counts of the matched (black) and unmatched (blue) cyclones. The median values for the 40-year dataset are given by the orange bars in the boxes and the boxes span the interquartile range. The whiskers extend to show the full range of the data. The box and whiskers for the matched and unmatched cyclones are slightly offset for clarity.

The plots in the right columns of Figs. 8 and 9 show, for the same times as the plots in the left columns, vertical crosssections in the cyclone motion direction through the centres of the composite cyclones. Relative vorticity is shown to indicate the strength and tilt of the cyclonic structure. Selected contours of PV indicate the dynamical tropopause (the 2-PVU surface) and regions of enhanced tropospheric PV, possibly caused by diabatic processes. Potential temperature contours indicate the change in static stability throughout the troposphere and can be compared to the $\theta_{900}^{\prime}$ field shown in the plots in the left columns. In contrast to the similarity of the evolution of the near-surface fields for the matched and unmatched cyclones, the vertical cross-sections show distinct characteristics. The composite cyclone evolves from a rearward (westward) tilted structure at the time of maximum growth rate to a more vertical structure at maximum intensity time in both composites. However, the tilt is more prominent at times prior to the maximum growth rate in the composite of unmatched cyclones. It is also striking that unmatched cyclones are dominated by lower-tropospheric $\xi$ at early times and the upper-tropospheric $\xi$ amplifies faster to become comparable to the low-level $\xi$ at time of maximum growth and maximum intensity. The region of enhanced $\xi$ also extends further northwards with height in both composites at the time of maximum growth rate, although the northwards distance of the peak $\xi$ is similar (not shown). This behaviour is consistent with baroclinic wave growth initiated from lower levels, akin to Pettersen type A mid-latitude cyclogenesis, where the tropopause-level anomaly is generated as a result of the baroclinic interaction with low levels. 
In the cyclones matched with TPVs, the $\xi$ at tropopause level is approximately equal to the low-level $\xi$ at the earliest time shown and it amplifies faster in the composites such that it dominates at the time of maximum growth rate. At maximum intensity, the cyclone core is stronger throughout the depth of the cyclone for the matched composite. Consistent with the stronger cyclonic $\xi$ at the tropopause in the matched cyclone composite, the tropopause extends down to higher pressures, reaching close to $550 \mathrm{hPa}$ at maximum intensity time. Thermal wind balance relates the vertical gradient in $\xi$ to the horizontal curvature in the $\theta$-anomaly field through the following equation:

$\frac{\partial \xi}{\partial z}=\frac{g}{\theta_{0}} \nabla^{2} \theta^{\prime}$

where $g$ is the acceleration due to gravity and $\theta_{0}$ is a reference $\theta$ value. At maximum intensity the upper- and lower-tropospheric features align to form a columnar vortex in which $\xi$ increases with height, $\nabla^{2} \theta^{\prime}>0$ and therefore a minimum in $\theta^{\prime}$ is expected on all pressure levels. In other words, the matched cases evolve to a cold-core vortex ( $\theta$-surfaces bow upwards) throughout the troposphere because their $\xi$ is dominated by the TPV at upper levels. The $\theta$-signature in the lower-troposphere is weak in the composite, but can be strong in individual cases. Enhanced PV values also extend throughout the troposphere at time of maximum intensity in both composites, but with larger values in the matched composite. Note that at one day before the maximum growth below $650 \mathrm{hPa}$ the PV tilts eastwards with height (opposite tilt to $\xi$ ). This is expected for baroclinic growth over height levels where the low-level thermal anomaly dominates the wind field in PV inversion (Methven et al., 2005). It arises because the meridional gradient of low-level $\theta$ is negative (as can be seen in the composite maps) while the background $\mathrm{PV}$ gradient is positive, so northward displacement of air is associated with lower boundary $\theta^{\prime}>0$ (inducing cyclonic flow) but a negative PV anomaly (inducing anticyclonic flow). By the time of maximum growth rate, the upper-tropospheric feature is dominant and large amplitude in the sense that the tropopause comes down low within the TPV, particularly in the matched composite. In this situation, the PV also tilts westwards with height. In both the matched and unmatched composites, there is a secondary maximum in PV near the ground and additionally for the matched composite, enhanced PV in the mid-troposphere. These features indicate the influence of diabatic and frictional processes since the development of a new maximum could not occur in conservative flow.

The evolution of the composite structure tilt in $\xi$, relative to the cyclone motion direction, is summarised for the matched and unmatched cyclones in Fig. 10. The tilt upshear (i.e., rearwards relative to the cyclone propagation direction) is greatest at time of maximum growth rate, the necessary configuration for baroclinic growth. The tilt is also rearwards at earlier times in both composites, although more strongly in the unmatched cases. As baroclinic waves grow the tilt is expected to tend towards a phase-locked configuration between tropopause-level and low-level counter-propagating Rossby waves-if the tilt is initially less than this then it will increase with time as the wave amplifies which is counter to the differential advection of the disturbances by the shear flow. This may explain the composite behaviour observed. However, it is also possible that the structures of systems averaged in the composite are most similar in their phase-locked configuration at time of maximum growth, and the weaker tilt and amplitude at earlier times is a result of averaging together systems with different structures. After the time of maximum growth, the tilt reduces markedly. However, despite this general similarity in evolution, there are differences between the two composites that are robust given the marked standard errors in the $\xi$ centre displacements. The 
https://doi.org/10.5194/wcd-2021-30

Preprint. Discussion started: 7 June 2021

(c) Author(s) 2021. CC BY 4.0 License.

Weather and

Climate Dynamics

Discussions

L.

matched cyclones acquire a maximum tilt distance (distance between the upper-tropospheric maximum in $\xi$ and that at $900 \mathrm{hPa}$ ) of $\approx 120 \mathrm{~km}$ compared to $\approx 160 \mathrm{~km}$ for the unmatched cyclones at the time of maximum growth rate. The matched cyclones also retain their tilted structure for a shorter time, aligning vertically (below $\sim 300 \mathrm{hPa}$ ) only 12 hours following maximum growth rate. These findings are consistent with the matched Arctic cyclones interacting with an isolated TPV and forming a single columnar vortex structure, while unmatched cyclones have rearward tilts at all stages, consistent with baroclinic wave growth through interaction between the low-level wave on the Arctic Frontal Zone and a tropopause-level disturbance.

\subsection{Comparison of Arctic and mid-latitude cyclone structure}

The structural evolution of these Arctic summer cyclones, as shown in Figs. 8-10, can be compared with that of mid-latitude winter cyclones, beginning with the near-surface structure. Dacre et al. (2012) generated a cyclone atlas by compositing the 200 most intense winter (December-February) North Atlantic cyclones from 1989 to 2009 using ERA-Interim data. Although a warm sector develops with a similar structure in the Arctic cyclones and to that found for the North Atlantic cyclones, the relatively cold core of the Arctic cyclones contrasts with the warm core typical of mid-latitude cyclones (see frontal locations in Fig. 3 of Dacre et al. (2012) and also low-level temperature composites generated using the associated cyclone atlas website). A relatively warm core at maturity is also consistent with the two predominant conceptual models of mid-latitude cyclones: the Norwegian cyclone model with occluded front wrapping to the north of the cyclone centre and the Shapiro-Keyser model with a warm air seclusion (Shapiro and Keyser, 1990). These summer Arctic cyclones are substantially weaker in terms of MSLP at their maximum intensity time than the winter mid-latitude cyclones (minimum MSLP of $\approx 990 \mathrm{hPa}$ compared to $<970 \mathrm{hPa}$ in the cyclone atlas). However, summer mid-latitude cyclones are also weaker than winter cyclones. For example, Čampa and Wernli (2012) found that the modal minimum sea level pressure for all tracked winter cyclones (also using ERAInterim data) was 970-990 hPa for cyclones in the regions of the Gulf of Alaska and between Greenland and Iceland in winter but $990-1010 \mathrm{~Pa}$ in summer.

The structural evolution of the $\xi$ cross-sections in the Arctic cyclone composites can also be compared to equivalent composites for winter mid-latitude cyclones generated using the cyclone atlas website which show the largest values of $\xi$ are present at near-surface levels one and two days prior to time of maximum intensity. This structure thus more closely resembles that for the unmatched than the matched cyclones. The vertically-coherent PV feature found in both cyclone composites was termed a "PV tower" by Rossa et al. (2000) and is characteristic of mature mid-latitude cyclones (Čampa and Wernli, 2012). Rossa et al. (2000) showed that the PV in the tower in a late autumn case study had three distinct origins: the top portion was stratospheric air in the region of lowered tropopause; the bottom portion was PV generated in the boundary layer by non-conservative diabatic processes (both friction and heating); and the middle portion was also generated by diabatic processes (chiefly latent heating in ascending air). Such PV towers can also occur in strong summer mid-latitude cyclones and Martínez-Alvarado et al. (2016) analysed strong diabatic contributions to the tower in such an event. In these Arctic composites the middle PV anomaly is much weaker than for mid-latitude cyclones, suggesting that latent heat release is less important to this structure than in mid-latitude cyclones. The tropopause-level structure in the matched cyclone composite can be compared to that of composite TPVs. Figure 9 of Cavallo et al. (2010) shows composite cyclonic TPV structures produced using 568 TPV samples simulated 
using the Weather Research and Forecasting (WRF) model with 30-km horizontal grid spacing and 31 vertical levels. The tropopause fold extends down to about $550 \mathrm{hPa}$, similar to the matched cyclone composite at maximum intensity time. A cold anomaly is present at and below the tropopause in both the TPV composite and matched cyclone composite. Additionally, the peak in the meridional wind speed at tropopause level in the TPV composite is consistent with the peak in $\xi$ at tropopause level in the matched composite.

Finally, the evolution of vorticity tilt in the Arctic cyclones can be compared that of mid-latitude cyclones. Figure 7 of Bengtsson et al. (2009) shows, calculated using the same methodology as used here, the tilt evolution of the 100 most intense cyclones found in the 40-year ECMWF reanalysis (ERA-40) and a 32-year, end of twentieth century, climate integration with the ECHAM5 model (though note that in Bengtsson et al. (2009) the 36 hours on either side of the maximum intensity time rather than the 48 hours on either side of the time of maximum growth rate are considered). The general increase in upstream tilt as the cyclones intensify, followed by a decrease to near vertical orientation at their time of maximum intensity is a trait shared by the mid-latitude and Arctic cyclone composites. However, in their mid-latitude composite, the maximum upstream tilt was $\approx 3-4^{\circ}$ great circle separation $(\approx 333-444 \mathrm{~km})$ in $\xi$ centres at 900 and $200 \mathrm{hPa}$, roughly coincident with the maximum growth rate time. Although this separation is more than double that found in our unmatched Arctic cyclone composite, the Arctic cyclones are typically much smaller scale. One reason is that the Rossby radius is at most $500 \mathrm{~km}$ for the Arctic cyclone environment, approximately half the value typical of mid-latitude systems. Some of this difference may also be attributable to the smaller, and so more extreme in intensity, sample used in Bengtsson et al. (2009) (the 100 rather than 200 most intense cyclones). However, this effect is likely to be somewhat compensated for by the compositing here relative to the time of maximum growth rate (when the tilt should be strongest) rather than time of maximum intensity (meaning that the cyclones contributing to Fig. 7 of Bengtsson et al. (2009) are unlikely to all have their maximum tilt at the same time). Nevertheless, for the Arctic cyclones matched with TPVs the upshear tilt is still considerably smaller than in mid-latitude cases, even as a proportion of cyclone scale.

\section{Conclusions}

Arctic cyclones are the major weather-related hazard in the Arctic. However, in comparison with mid-latitude cyclones there have been very few studies examining their structure, evolution and mechanisms for growth. Here the focus has been on summer-time Arctic cyclones because human activity in the Arctic is greatest in this season and because they play a major role in modifying the sea ice distribution over the marginal ice zone where ice fraction is less than one. Arctic cyclones in summer also typically have much larger scale than the intense polar lows that occur in winter. Case studies of Arctic cyclones in summer have focused on the most intense or long-lived examples. Most studies (see Sect. 1) refer to interaction with a tropopause polar vortex (TPV) disturbance and imply that such disturbances are important to the growth of the Arctic cyclone. However, this link has not been explored taking into account a more complete climatological set of Arctic cyclone and TPV events.

The chief purpose of this paper is to characterise and quantify the proportion of Arctic cyclones that are near to a TPV at the time of maximum cyclone growth rate through to maximum intensity (termed matched cyclones), and to examine the average 
https://doi.org/10.5194/wcd-2021-30

Preprint. Discussion started: 7 June 2021

(c) Author(s) 2021. CC BY 4.0 License.

Weather and

Climate Dynamics

Discussions

L

structure in matched and unmatched cases using a statistical composite approach. To the authors' knowledge, this is the first paper in which both low-level Arctic cyclones and TPVs have been tracked using the same tracking algorithm and reanalysis dataset (and the first use of ERA5 for such tracking), enabling statistics of genesis and tracks and to be compared systematically. Furthermore, the ERA5 data have been used to create dynamically consistent composites of the three-dimensional Arctic cyclone structures from the surface to the tropopause region enabling quantification of system tilt and deductions of the nature of the growth mechanisms.

The first research question was to determine the characteristics of TPVs and how they compare to those of Arctic cyclones. The locations of enhanced genesis and track densities of Arctic cyclones and TPVs were shown to be consistent with previous climatologies produced independently using other datasets (Cavallo et al. (2009) and Vessey et al. (2020), respectively). The frequency distributions of lifetimes and intensities were then calculated for the tracked Arctic cyclones and TPVs and also split into those with genesis within and outside the Arctic. While the modal lifetime of Arctic cyclones of 2-3 days exceeds that of TPVs, TPVs have a longer mean lifetime (5.0 compared to 4.4 days) skewed by the much longer tail of the TPV lifetime distribution (the longest TPV track was 43 days). The lifetime and intensity characteristics also depend on the genesis location of the features. Cyclones that track into the Arctic have longer mean lifetimes and are also more intense on average than those that have their genesis within the Arctic. In contrast, TPVs with genesis within the Arctic are much more frequent and more intense on average than those with genesis further south. The annual counts of Arctic cyclones and TPVs are significantly correlated at the $95 \%$ level for features with both Arctic genesis and non-Arctic genesis suggesting that TPVs have a role in the initiation and/or intensification of Arctic cyclones.

505 The second research question addressed the role of TPVs in the initiation and intensification of Arctic cyclones. The percentage of Arctic cyclones matched with a TPV (varying the great circle separation criterion in the range $1-5^{\circ}$ ) increases from $1-10 \%$ at the genesis time of Arctic cyclones to 9-35\% at the time of maximum growth rate and 9-38\% at maximum intensity. Hence, even with the greatest separation criterion (approximately equal to the Rossby radius) the majority of Arctic cyclones (about two thirds) are developing without a nearby TPV. Also these statistics suggest that TPVs do not play a part in the genesis of most Arctic cyclones, but have a potential role in subsequent Arctic cyclone growth. The geographical distribution of matched and unmatched cyclone cases also differs markedly. Matched cyclones preferentially track over the Arctic Ocean to the north of the Alaskan and Canadian coastline, and the Canadian Archipelago. In contrast, unmatched cyclones track preferentially over the Barents and Kara Seas to the north of the Russian coastline. TPV track density is higher across the North American Arctic coastline and Canadian Archipelago region, accounting for the higher proportion of matched Arctic cyclone cases in this area. The unmatched cyclone cases have a higher genesis frequency over northern Eurasia, both within the Arctic region and south to approximately $55^{\circ} \mathrm{N}$, associated with the strong low level baroclinicity, especially along the Arctic Frontal Zone associated with the Arctic Ocean coastline in summer. There is a general tendency for the number of matched cyclones to decrease to a minimum in August of the extended May-September summer season with a corresponding increase in the number of unmatched cyclones.

520 Finally, the third research question addressed how the evolution of Arctic cyclone structure and intensity is modified by interaction with TPVs. The matched and unmatched cyclones were compared by compositing the 200 most intense cyclones 
https://doi.org/10.5194/wcd-2021-30

Preprint. Discussion started: 7 June 2021

(c) Author(s) 2021. CC BY 4.0 License.

Weather and

Climate Dynamics

Discussions

L

from each cyclone set at four specific times, tracing back the structural evolution: the time of Arctic cyclone maximum intensity, maximum growth rate, then one and two days prior to maximum growth rate. The two composite sets revealed distinct structural evolution and the three-dimensional structure of dynamical fields was used to deduce the relative importance of mechanisms acting in each set.

The unmatched Arctic cyclones have a larger amplitude low-level $\theta$-wave on average and develop a more pronounced warm sector by the time of maximum growth rate. The $\xi$ is dominated by low levels two days prior to maximum growth rate and then upper-tropospheric $\xi$ grows faster so that it attains a similar magnitude near the tropopause and surface with a pronounced upshear tilt with height (i.e., rearwards relative to the cyclone motion vector) which is strongest at time of maximum growth rate. There is no pre-existing (tracked) tropopause disturbance and the anomaly here grows in these two days as a result of the mutual growth with the lower wave. These structural features are all commensurate with the baroclinic growth mechanism dominated by a low-level disturbance on a baroclinic zone at initial time: type A cyclogenesis. Between the time of maximum growth rate and maximum intensity the tilt reduces, but remains upshear on average. A PV tower does not develop because there is a distinct mid-level gap in PV between the tropopause-level PV anomaly and positive PV anomaly near the boundary layer. These features indicate that latent heat release is not as important in this type of cyclone compared with typical midlatitude cases. Finally, the track density map for unmatched cases shows how they preferentially track along the north coast of Russia, but the genesis can be further south, associated with crests of waves on the strong baroclinic zone there.

In contrast, the matched Arctic cyclones are upper-troposphere dominated in the upshear-tilted baroclinic configuration at the time of maximum growth rate. This upper-tropospheric vorticity disturbance is identified with the tracked TPV in these cases. The tropopause is markedly lower in association with the upper PV anomaly in this composite (down to about $550 \mathrm{hPa}$ compared to about $475 \mathrm{hPa}$ for the unmatched composite). Interestingly, two days before maximum growth rate the upper and lower $\xi$ are equally weighted in the composite, the $\xi$ tilts slightly westwards and the PV tilts eastwards in the lower troposphere. These are all features consistent with a dry baroclinic growth mechanism through cooperative interaction between an tropopause-level PV disturbance and lower-boundary $\theta$-wave. However, the upper disturbance is so dominant by the time of maximum growth that the PV tilts westwards at all levels. By the time of maximum intensity, there is some enhancement of mid-level PV as well as the TPV and boundary layer PV anomalies, indicating some influence of latent heat release.

The matched cyclone composite has some features that differ markedly from mid-latitude composite structures. The upshear tilt is relatively weak, even at the time of maximum growth rate. There is no detectable tilt on average at the time of maximum intensity: the structure forms a single columnar vortex. The $\xi$ is upper-tropospheric dominated and therefore thermal wind balance implies that it must have a cold core and $\theta$-surfaces bow upwards in the centre of the cyclone. The structure is very similar to the wind and thermal structure obtained by inversion of an isolated tropopause-level PV anomaly using a balance approximation (e.g., see Fig. 1 in Thorpe, 1986) and characteristic of the structure of isolated TPVs (Cavallo et al., 2010). Near the lower boundary, a warm sector forms to the southeast of the cyclone centre (assuming eastwards cyclone motion), while the cold air wraps around the composite cyclone centre to the northwest, but the dominance of the upper-level PV results in a cold core surface at maturity. This contrasts with the composite structure of intense mid-latitude cyclones which tend to form a warm seclusion at low levels in their mature phase and are therefore warm core in the lower troposphere, even though they are 
cold core in the upper troposphere. For example, Martínez-Alvarado et al. (2014) show a dropsonde curtain from the centre of an intense cyclone of the Shapiro-Keyser type through the bent-back warm front that arcs round the warm seclusion. The front slopes outwards with height where $\theta$-surfaces dip steeply downwards towards the cyclone centre in the lower troposphere.

One plausible explanation for the behaviour in the matched Arctic cyclone cases is that they develop away from a strong baroclinic zone and associated jet stream. The final stages of growth arise from the baroclinic interaction of the pre-existing TPV, with its strong PV anomaly on the tropopause, with a low-level cyclone that has already formed. Theoretical studies based on quasi-geostrophic dynamics have shown the tendency for upper and lower vortices to approach and align one above the other (Polvani, 1991) or for isolated tilted vortex disturbances to become upright (Reasor and Montgomery, 2001). Such behaviour is prohibited if the large-scale vertical shear is too strong.

In summary, this research has demonstrated that TPVs are in close proximity (within one Rossby radius) of Arctic cyclones at their time of maximum intensification rate in about one third of cases. TPVs are far less likely to be within range at the initial cyclogenesis. However, more Arctic cyclones do tend to occur in summers with increased numbers of TPVs. The track density pattern of Arctic cyclones matched with TPVs is geographically distinct from that of unmatched cyclones: Arctic cyclones that are associated with a TPV during their intensification stage preferentially track along the North American shore of the Arctic Ocean and the Canadian Archipelago, whereas unmatched cyclone tracks are much more frequent along the Eurasian shore of the Arctic Ocean. The cyclones in matched cases are dominated by the flow associated with the upper-level $\mathrm{PV}$ and develop from upper-level precursors, while the cyclones in the unmatched cases grow from low-level warm anomalies extending polewards on the Arctic Frontal Zone and the upper-level PV grows rapidly as a result of advection by the winds associated with the low-level disturbance during baroclinic growth. Therefore, the unmatched cases have more similarity with mid-latitude cyclone dynamics, while the Arctic cyclones matched with TPVs more closely resemble the multi-layer interaction between isolated vortices.

Code and data availability. ERA5 data are freely available and downloadable from the Copernicus Climate Data Store (https://cds.climate.copernicus.eu). The TRACK algorithm is available on the University of Reading's Git repository (GitLab) at https://gitlab.act.reading.ac.uk/public. plotting code. JLV performed preliminary analysis as part of his M.Sc. dissertation supervised by the other authors and further analysis was performed by the other authors after the dissertation was completed. SLG drafted the paper and produced the final plots. The other authors provided edits to the paper.

Competing interests. No competing interests are present 
https://doi.org/10.5194/wcd-2021-30

Preprint. Discussion started: 7 June 2021

(c) Author(s) 2021. CC BY 4.0 License.

Weather and

Climate Dynamics

(c) (i)

Discussions

L

585 Acknowledgements. The authors acknowledge the European Centre for Medium Range Forecasting (ECMWF) for the production of ERA5 dataset. The results contain modified Copernicus Climate Change Service information [1979-2018]. Hersbach et al. (2018b) and Hersbach et al. (2018a) were downloaded from the Copernicus Climate Change Service (C3S) Climate Data Store. Neither the European Commission nor ECMWF is responsible for any use that may be made of the Copernicus information or data it contains. KIH's contribution was funded by the United Kingdom's Natural Environment Research Council (NERC) as part of the National Centre for Atmospheric Sciences. 
(a)
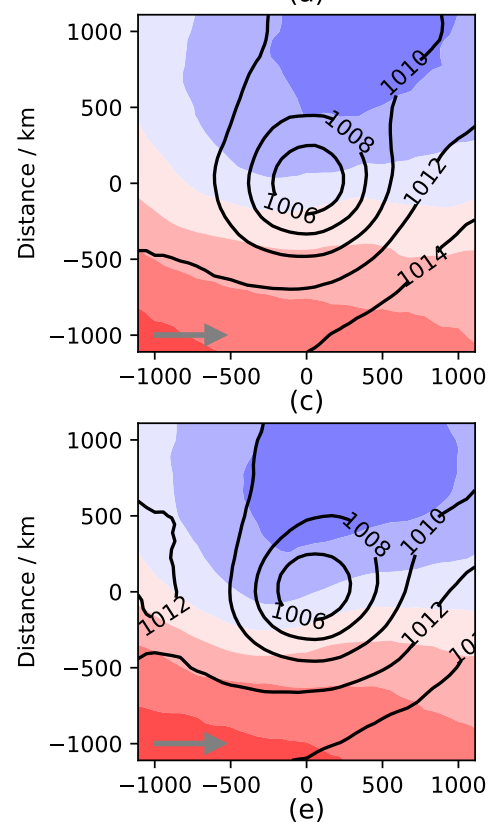

(e)

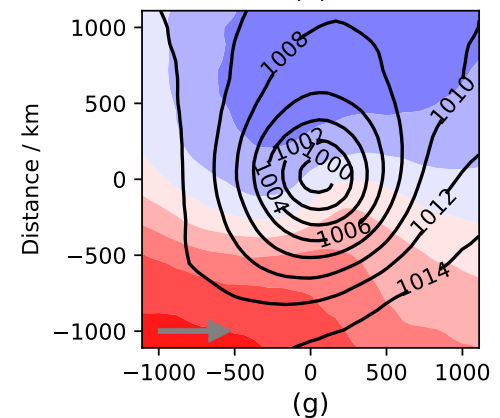

(g)

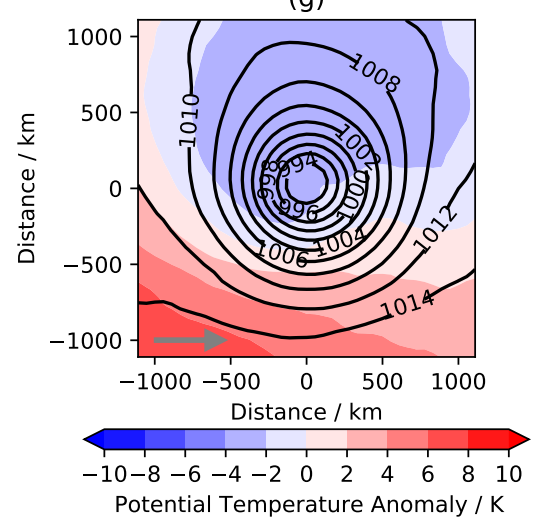

(b)

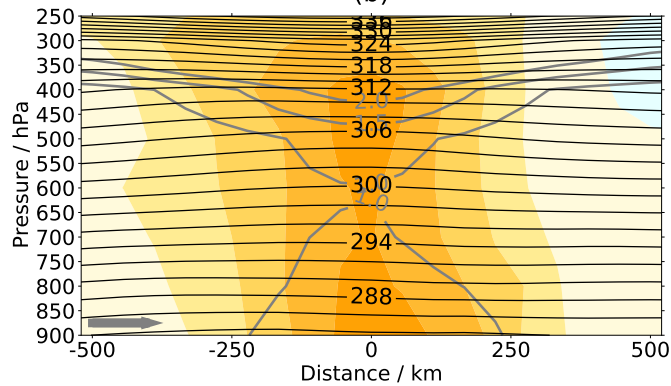

(d)

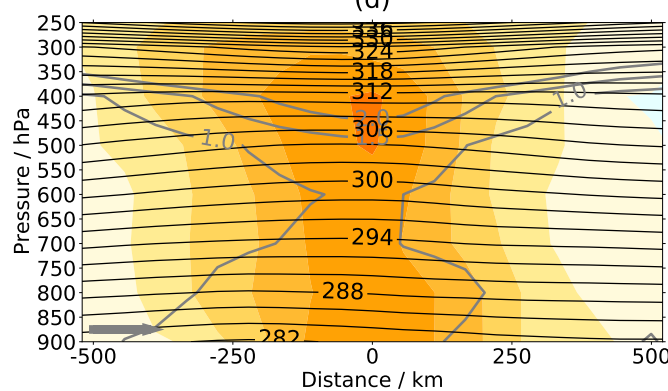

(f)

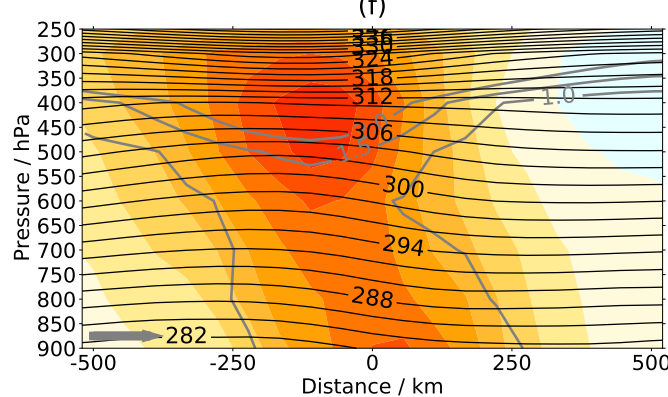

(h)

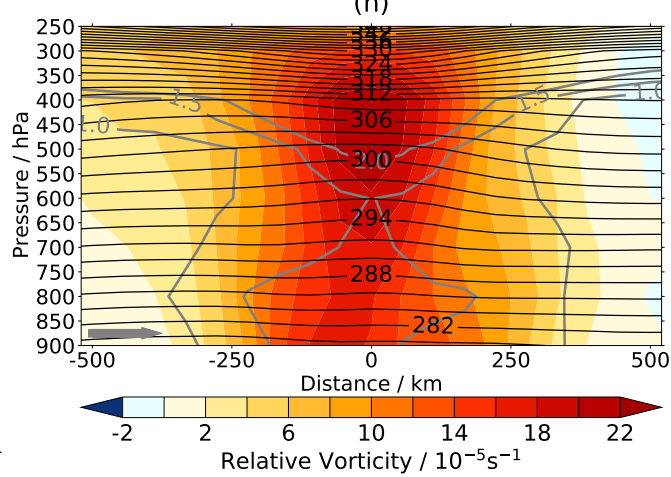

Figure 8. Composite fields of the 200 most intense matched Arctic cyclones at times (a,b) two days prior to maximum growth rate, (c,d) one day prior to maximum growth rate, $(\mathrm{e}, \mathrm{f})$ maximum growth rate, $(\mathrm{g}, \mathrm{h})$ maximum intensity. Left column shows the horizontal distributions of $\theta_{900}$ anomalies relative to the domain average (shaded) and MSLP (hPa). Right column shows vertical cross-sections of $\xi$ in the along-track direction (shaded). Thick, grey contours: potential vorticity (1, 1.5 and 2 PVU shown). Black contours: $\theta$ (interval $2 \mathrm{~K}$ ). Grey arrow in each panel indicates direction of cyclone motion. The composites use full resolution (rather than filtered) fields. 
https://doi.org/10.5194/wcd-2021-30

Preprint. Discussion started: 7 June 2021

(c) Author(s) 2021. CC BY 4.0 License.

Weather and

Climate Dynamics

Discussions
L. (a)
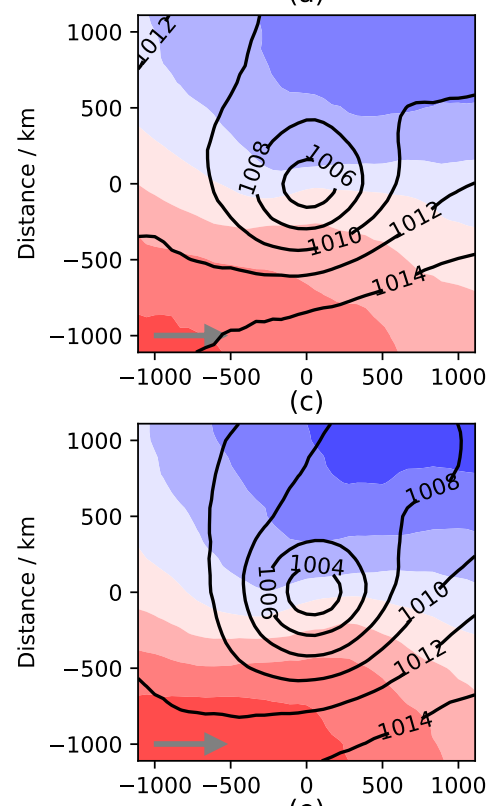

(e)

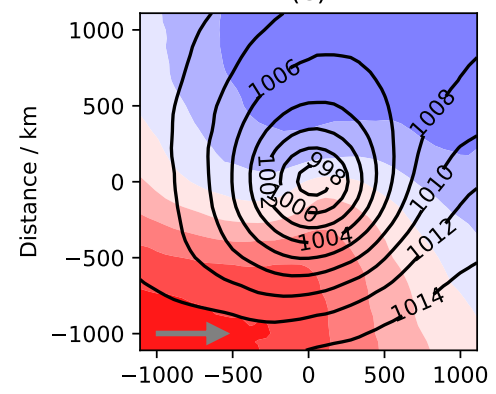

(g)

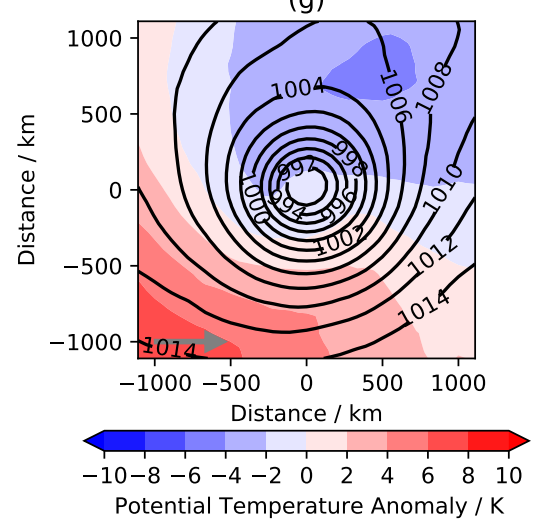

(b)

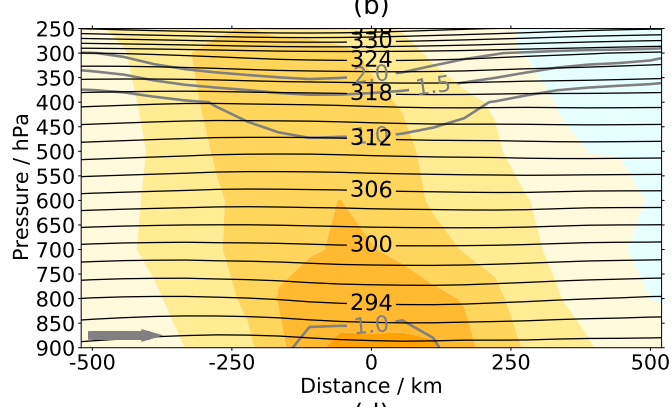

(d)

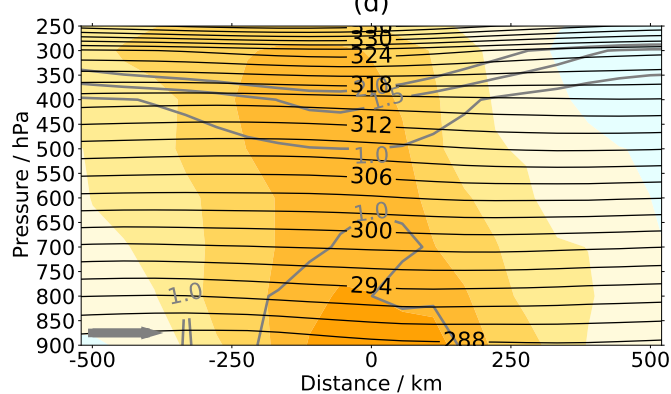

(f)

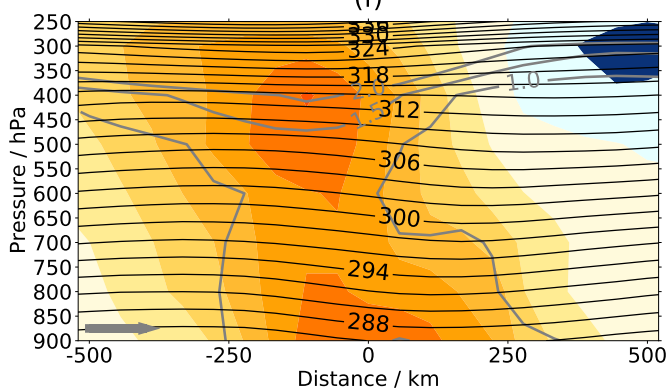

(h)

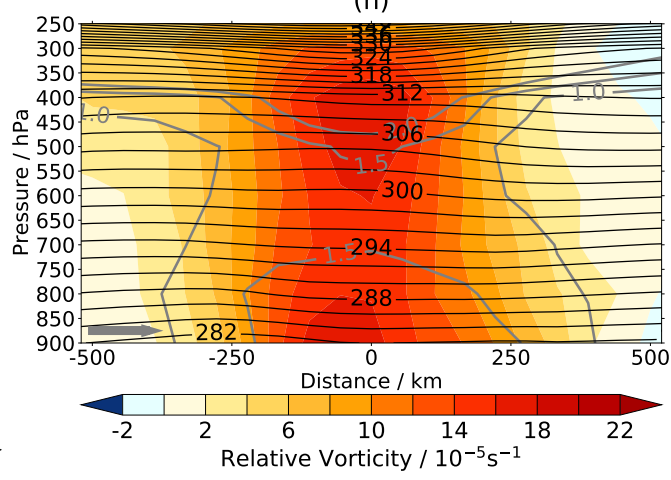

Figure 9. As for Fig. 8 but for the 200 most intense unmatched Arctic cyclones. 
https://doi.org/10.5194/wcd-2021-30

Preprint. Discussion started: 7 June 2021

(c) Author(s) 2021. CC BY 4.0 License.

(c) (i)

Weather and

Climate Dynamics

Discussions (a)

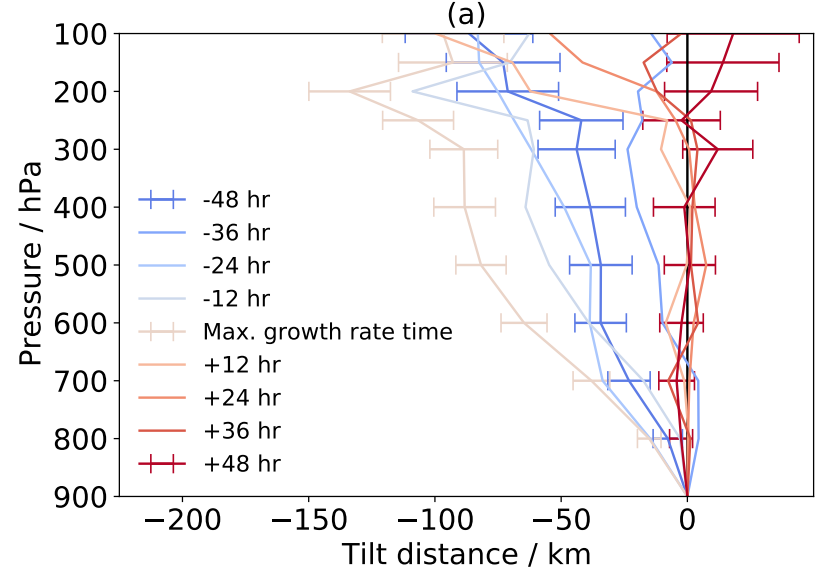

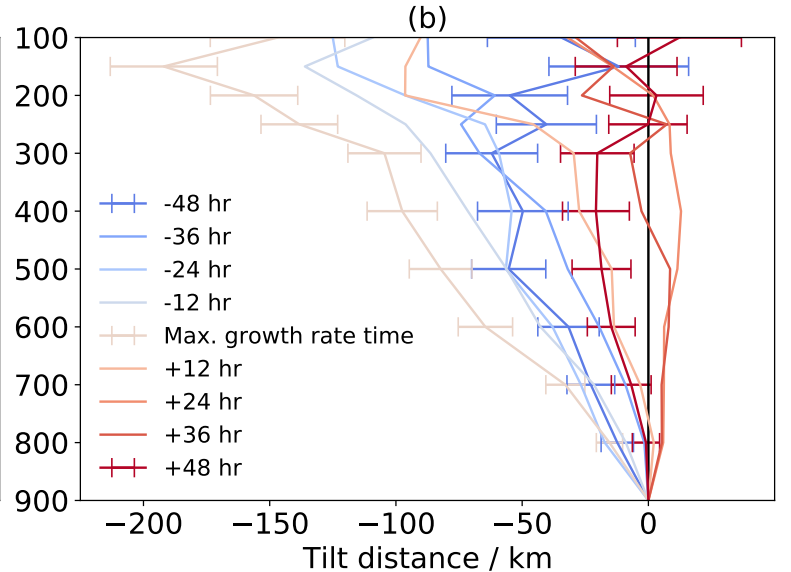

Figure 10. Evolution of composite vorticity tilt structure from 48 hours before to 48 hours after the time of maximum growth rate for the 200 most intense (a) matched and (b) unmatched cyclones. Illustrative standard error bars are shown at three times. Tilt distance is the great circle distance relative to the $\xi$ centre at $900 \mathrm{hPa}$ 
https://doi.org/10.5194/wcd-2021-30

Preprint. Discussion started: 7 June 2021

(c) Author(s) 2021. CC BY 4.0 License.

Weather and

Climate Dynamics

(c)

\section{References}

Bengtsson, L., Hodges, K. I., and Keenlyside, N.: Will Extratropical Storms Intensify in a Warmer Climate?, J. Climate, 22, 2276-2301, https://doi.org/10.1175/2008JCLI2678.1, 2009.

Čampa, J. and Wernli, H.: A PV Perspective on the Vertical Structure of Mature Midlatitude Cyclones in the Northern Hemisphere, J. Atmos. Sci., 69, 725-740, https://doi.org/10.1175/JAS-D-11-050.1, 2012.

Cavallo, S. M. and Hakim, G. J.: Physical Mechanisms of Tropopause Polar Vortex Intensity Change, J. Atmos. Sci., 70, 3359-3373, https://doi.org/10.1175/JAS-D-13-088.1, 2013.

Cavallo, S. M., Hakim, G. J., Cavallo, S. M., and Hakim, G. J.: Potential Vorticity Diagnosis of a Tropopause Polar Cyclone, Mon. Weather Rev., 137, 1358-1371, https://doi.org/10.1175/2008MWR2670.1, 2009.

Cavallo, S. M., Hakim, G. J., Cavallo, S. M., and Hakim, G. J.: Composite Structure of Tropopause Polar Cyclones, Mon. Weather Rev., 138, 3840-3857, https://doi.org/10.1175/2010MWR3371.1, 2010.

Dacre, H. F., Hawcroft, M. K., Stringer, M. A., and Hodges, K. I.: An Extratropical Cyclone Atlas: A Tool for Illustrating Cyclone Structure and Evolution Characteristics, Bull. Amer. Meteorol. Soc., 93, 1497-1502, https://doi.org/10.1175/BAMS-D-11-00164.1, 2012.

Day, J. J. and Hodges, K. I.: Growing Land-Sea Temperature Contrast and the Intensification of Arctic Cyclones, Geophys. Res. Lett., 45, 3673-3681, https://doi.org/10.1029/2018GL077587, 2018.

Day, J. J., Hawkins, E., and Tietsche, S.: Will Arctic sea ice thickness initialization improve seasonal forecast skill?, Geophys. Res. Lett., 41, 7566-7575, https://doi.org/10.1002/2014GL061694, 2014.

Deveson, A. C. L., Browning, K. A., and Hewson, T. D.: A classification of FASTEX cyclones using a height-attributable quasi-geostrophic vertical-motion diagnostic, Quart. J. Roy. Meteorol. Soc., 128, 93-117, https://doi.org/https://doi.org/10.1256/00359000260498806, 2002.

Finocchio, P. M., Doyle, J. D., Stern, D. P., and Fearon, M. G.: Short-term Impacts of Arctic Summer Cyclones on Sea Ice Extent in the Marginal Ice Zone, Geophys. Res. Lett., 47, e2020GL088 338, https://doi.org/https://doi.org/10.1029/2020GL088338, 2020.

Gray, S. L. and Dacre, H. F.: Classifying dynamical forcing mechanisms using a climatology of extratropical cyclones, Quart. J. Roy. Meteorol. Soc., 132, 1119-1137, https://doi.org/https://doi.org/10.1256/qj.05.69, 2006.

Hakim, G. J. and Canavan, A. K.: Observed Cyclone-Anticyclone Tropopause Vortex Asymmetries, J. Atmos. Sci., 62, 231-240, https://doi.org/10.1175/JAS-3353.1, 2005.

Hersbach, H., Bell, B., Berrisford, P., Biavati, G., Horányi, A., Muñoz Sabater, J., Nicolas, J., Peubey, C., Radu, R., Rozum, I., Schepers, D., Simmons, A., Soci, C., Dee, D., and Thépaut, J.-N.: ERA5 hourly data on single levels from 1979 to present. Copernicus Climate Change Service (C3S) Climate Data Store (CDS). [Online] (Accessed between 04/2019-04/2021), https://doi.org/10.24381/cds.adbb2d47, 2018a.

Hersbach, H., Bell, B., Berrisford, P., Biavati, G., Horányi, A., Muñoz Sabater, J., Nicolas, J., Peubey, C.and Radu, R., Rozum, I., Schepers, D., Simmons, A., Soci, C., Dee, D., and Thépaut, J.-N.: ERA5 hourly data on pressure levels from 1979 to present. Copernicus Climate Change Service (C3S) Climate Data Store (CDS) [online]. (Accessed between 04/2019-04/2021), https://doi.org/10.24381/cds.bd0915c6, 2018 b.

Hersbach, H., Bell, B., Berrisford, P., Hirahara, S., Horányi, A., Muñoz-Sabater, J., Nicolas, J., Peubey, C., Radu, R., Schepers, D., Simmons, A., Soci, C., Abdalla, S., Abellan, X., Balsamo, G., Bechtold, P., Biavati, G., Bidlot, J., Bonavita, M., De Chiara, G., Dahlgren, P., Dee, D., Diamantakis, M., Dragani, R., Flemming, J., Forbes, R., Fuentes, M., Geer, A., Haimberger, L., Healy, S., Hogan, R. J., Hólm, E., Janisková, M., Keeley, S., Laloyaux, P., Lopez, P., Lupu, C., Radnoti, G., de Rosnay, P., Rozum, I., Vamborg, F., Villaume, S., and Thépaut, J.-N.: The ERA5 global reanalysis, Quart. J. Roy. Meteorol. Soc., 146, 1999-2049, https://doi.org/https://doi.org/10.1002/qj.3803, 2020. 
https://doi.org/10.5194/wcd-2021-30

Preprint. Discussion started: 7 June 2021

(c) Author(s) 2021. CC BY 4.0 License.

Weather and

Climate Dynamics

(c) (i)

Hodges, K. I.: A General Method for Tracking Analysis and Its Application to Meteorological Data, Mon. Weather Rev., 122, 2573-2586, https://doi.org/10.1175/1520-0493(1994)122<2573:AGMFTA>2.0.CO;2, 1994.

Hodges, K. I.: Feature Tracking on the Unit Sphere, Mon. Weather Rev., 123, 3458-3465, https://doi.org/10.1175/15200493(1995)123<3458:FTOTUS>2.0.CO;2, 1995.

Hodges, K. I.: Adaptive Constraints for Feature Tracking, Mon. Weather Rev., 127, 1362-1373, https://doi.org/10.1175/15200493(1999)127<1362:ACFFT>2.0.CO;2, 1999.

Hoskins, B. J., McIntyre, M. E., and Robertson, A. W.: On the use and significance of isentropic potential vorticity maps, Quart. J. Roy. Meteorol. Soc., 111, 877-946, https://doi.org/10.1002/qj.49711147002, 1985.

Jung, T. and Matsueda, M.: Verification of global numerical weather forecasting systems in polar regions using TIGGE data, Quart. J. Roy. Meteorol. Soc., 142, 574-582, https://doi.org/https://doi.org/10.1002/qj.2437, 2016.

Martínez-Alvarado, O., Baker, L., Gray, S., Methven, J., and Plant, R.: Distinguishing the cold conveyor belt and sting jet air streams in an intense extratropical cyclone, Mon. Weather Rev., 142, 2571-2595, https://doi.org/10.1175/MWR-D-13-00348.1, 2014.

Martínez-Alvarado, O., Gray, S. L., and Methven, J.: Diabatic Processes and the Evolution of Two Contrasting Summer Extratropical Cyclones, Mon. Weather Rev., 144, 3251-3276, https://doi.org/10.1175/MWR-D-15-0395.1, 2016.

Methven, J., Heifetz, E., Hoskins, B., and Bishop, C.: The counter-propagating Rossby wave perspective on baroclinic instability. Part III: Primitive equation disturbances on the sphere, Quart. J. Roy. Meteorol. Soc., 131, 1393-1424, 2005.

National Weather Service Climate Prediction Center: North Atlantic Oscillation (NAO) Historical Index. [Online]. Accessed on 12 May, 2021, https://www.cpc.ncep.noaa.gov/data/teledoc/nao.shtml, 2021.

645 Neu, U., Akperov, M. G., Bellenbaum, N., Benestad, R., Blender, R., Caballero, R., Cocozza, A., Dacre, H. F., Feng, Y., Fraedrich, K., Grieger, J., Gulev, S., Hanley, J., Hewson, T., Inatsu, M., Keay, K., Kew, S. F., Kindem, I., Leckebusch, G. C., Liberato, M. L. R., Lionello, P., Mokhov, I. I., Pinto, J. G., Raible, C. C., Reale, M., Rudeva, I., Schuster, M., Simmonds, I., Sinclair, M., Sprenger, M., Tilinina, N. D., Trigo, I. F., Ulbrich, S., Ulbrich, U., Wang, X. L., and Wernli, H.: IMILAST: A Community Effort to Intercompare Extratropical Cyclone Detection and Tracking Algorithms:, Bull. Amer. Meteorol. Soc., 94, 529-547, https://doi.org/10.1175/BAMS-D-11-00154.1, 2013.

Overland, J. E. and Wang, M.: When will the summer Arctic be nearly sea ice free?, Geophys. Res. Lett., 40, 2097-2101, https://doi.org/10.1002/grl.50316, 2013.

Petterssen, S. and Smebye, S. J.: On the development of extratropical cyclones, Quart. J. Roy. Meteorol. Soc., 97, 457-482, https://doi.org/10.1002/qj.49709741407, 1971.

Polvani, L.: Two-layer geostrophic vortex dynamics. Part 2. Alignment and two-layer V-states, J. Fluid Mech., 225, 241-270, https://doi.org/10.1017/S0022112091002045, 1991.

Reasor, P. and Montgomery, M.: Three-dimensional alignment and co-rotation of weak, TC-like vortices via linear vortex Rossby waves, J. Atmos. Sci., 58, 2306-2330, https://doi.org/10.1175/1520-0469(2001)058<2306:TDAACO>2.0.CO;2, 2001.

Rossa, A. M., Wernli, H., and Davies, H. C.: Growth and Decay of an Extra-Tropical Cyclone's PV-Tower, Meteorol. Atmos. Phys., 73, 139-156, https://doi.org/10.1007/s007030050070, 2000.

Schreiber, E. A. P. and Serreze, M. C.: Impacts of synoptic-scale cyclones on Arctic sea-ice concentration: a systematic analysis, Ann. Glaciol., 61, 139-153, https://doi.org/10.1017/aog.2020.23, 2020.

Shapiro, M. and Keyser, D.: Fronts, jet streams, and the tropopause, in: Extratropical cyclones, the Erik Pálmen memorial volume, edited by Newton, C. W. and Holopainen, E. O., pp. 167-191, Amer. Meteor. Soc., 1990. 
https://doi.org/10.5194/wcd-2021-30

Preprint. Discussion started: 7 June 2021

(c) Author(s) 2021. CC BY 4.0 License.

(c) (i)

Weather and

Climate Dynamics

Discussions

Simmonds, I. and Rudeva, I.: The great Arctic cyclone of August 2012, Geophys. Res. Lett., 39, L23709, https://doi.org/10.1029/2012GL054259, 2012.

Simmonds, I. and Rudeva, I.: A comparison of tracking methods for extreme cyclones in the Arctic basin, Tellus, 66, 25 252, https://doi.org/10.3402/tellusa.v66.25252, 2014.

Stephenson, S. R., Smith, L. C., Brigham, L. W., and Agnew, J. A.: Projected 21st-century changes to Arctic marine access, Climatic Change, 118, 885-899, https://doi.org/10.1007/s10584-012-0685-0, 2013.

670 Tanaka, H., Yamagami, A., and Takahashi, S.: The structure and behavior of the arctic cyclone in summer analyzed by the JRA-25/JCDAS data, Polar Science, 6, 55-69, https://doi.org/https://doi.org/10.1016/j.polar.2012.03.001, special Issue: The Second International Symposium on the Arctic Research (ISAR - 2), 2012.

Tao, W., Zhang, J., Fu, Y., and Zhang, X.: Driving Roles of Tropospheric and Stratospheric Thermal Anomalies in Intensification and Persistence of the Arctic Superstorm in 2012, Geophys. Res. Lett., 44, 10,017-10,025, https://doi.org/10.1002/2017GL074778, 2017.

Thorpe, A. J.: Synoptic Scale Disturbances with Circular Symmetry, Mon. Weather Rev., 114, 1384-1389, https://doi.org/10.1175/15200493(1986)114<1384:SSDWCS>2.0.CO;2, 1986.

Tilinina, N., Gulev, S. K., and Bromwich, D. H.: New view of Arctic cyclone activity from the Arctic system reanalysis, Geophys. Res. Lett., 41, 1766-1772, https://doi.org/10.1002/2013GL058924, 2014.

Vessey, A. F., Hodges, K. I., Shaffrey, L. C., and Day, J. J.: An inter-comparison of Arctic synoptic scale storms between four global reanalysis datasets, Clim. Dynam., 54, 2777-2795, https://doi.org/10.1007/s00382-020-05142-4, 2020.

Wickström, S., Jonassen, M. O., Vihma, T., and Uotila, P.: Trends in cyclones in the high-latitude North Atlantic during 1979-2016, Quart. J. Roy. Meteorol. Soc., 146, 762-779, https://doi.org/https://doi.org/10.1002/qj.3707, 2020.

Yamagami, A., Matsueda, M., and Tanaka, H. L.: Extreme Arctic cyclone in August 2016, Atmos. Sci. Lett., 18, 307-314, https://doi.org/10.1002/asl.757, 2017.

Yamagami, A., Matsueda, M., and Tanaka, H. L.: Predictability of the 2012 Great Arctic Cyclone on medium-range timescales, Polar Science, 15, 13-23, https://doi.org/10.1016/J.POLAR.2018.01.002, 2018a.

Yamagami, A., Matsueda, M., and Tanaka, H. L.: Medium-Range Forecast Skill for Extraordinary Arctic Cyclones in Summer of 2008-2016, Geophys. Res. Lett., 45, 4429-4437, https://doi.org/10.1029/2018GL077278, 2018 b.

Yamazaki, A., Inoue, J., Dethloff, K., Maturilli, M., and König-Langlo, G.: Impact of radiosonde observations on forecasting summertime Arctic cyclone formation, J. Geophys. Res., 120, 3249-3273, https://doi.org/10.1002/2014JD022925, 2015.

Zhang, X., Walsh, J. E., Zhang, J., Bhatt, U. S., and Ikeda, M.: Climatology and interannual variability of Arctic cyclone activity: 1948-2002, J. Climate, 17, 2300-2317, https://doi.org/10.1175/1520-0442(2004)017<2300:CAIVOA>2.0.CO;2, 2004. 